\title{
A systems immunology approach identifies the collective impact of 5 miRs in Th2 inflammation
}

\author{
Ayşe Kılıç, ${ }^{1}$ Marc Santolini, ${ }^{2,3,4}$ Taiji Nakano, ${ }^{1}$ Matthias Schiller, ${ }^{5}$ Mizue Teranishi,, ${ }^{6}$ ascal Gellert, ${ }^{7}$ \\ Yuliya Ponomareva, ${ }^{8}$ Thomas Braun, ${ }^{6}$ Shizuka Uchida, ${ }^{9}$ Scott T. Weiss, ${ }^{3}$ Amitabh Sharma, ${ }^{3}$ \\ and Harald Renz ${ }^{1}$ \\ IInstitute of Laboratory Medicine and Pathobiochemistry, Molecular Diagnostics, Philipps University Marburg, \\ Marburg, Germany. ${ }^{2}$ Center for Complex Network Research, Department of Physics, Northeastern University, Boston, \\ Massachusetts, USA. 'Brigham and Women's Hospital, Channing Division of Network Medicine, Department of Medicine, \\ Harvard Medical School, Boston, Massachusetts, USA. ${ }^{4}$ Center for Cancer Systems Biology (CCSB) and Department of \\ Cancer Biology, Dana-Farber Cancer Institute, Boston, Massachusetts, USA. ${ }^{5}$ Clinic for Dermatology and Venereology, \\ University Medical Center Rostock, Rostock, Germany. ${ }^{6}$ Max Planck Institute for Heart and Lung Research, Bad Nauheim, \\ Germany. ${ }^{7}$ Breast Cancer Now Research Centre at The Institute of Cancer Research, London, United Kingdom. ${ }^{8}$ Institute \\ of Cardiovascular Regeneration, Centre for Molecular Medicine, Goethe- University Frankfurt, Frankfurt Germany. \\ ${ }^{9}$ Cardiovascular Innovation Institute, University of Louisville, Louisville, Kentucky, USA.
}

\begin{abstract}
Allergic asthma is a chronic inflammatory disease dominated by a CD4 ${ }^{+}$T helper 2 (Th2) cell signature. The immune response amplifies in self-enforcing loops, promoting Th2-driven cellular immunity and leaving the host unable to terminate inflammation. Posttranscriptional mechanisms, including microRNAs (miRs), are pivotal in maintaining immune homeostasis. Since an altered expression of various miRs has been associated with $\mathrm{T}$ cell-driven diseases, including asthma, we hypothesized that miRs control mechanisms ensuring Th2 stability and maintenance in the lung. We isolated murine $\mathrm{CD} 4{ }^{+}$Th2 cells from allergic inflamed lungs and profiled gene and $\mathrm{miR}$ expression. Instead of focusing on the magnitude of miR differential expression, here we addressed the secondary consequences for the set of molecular interactions in the cell, the interactome. We developed the Impact of Differential Expression Across Layers, a network-based algorithm to prioritize disease-relevant miRs based on the central role of their targets in the molecular interactome. This method identified 5 Th2-related miRs (mir27b, mir206, mir106b, mir203, and mir23b) whose antagonization led to a sharp reduction of the Th2 phenotype. Overall, a systems biology tool was developed and validated, highlighting the role of miRs in Th2-driven immune response. This result offers potentially novel approaches for therapeutic interventions.
\end{abstract}

Authorship note: A. Sharma and $\mathrm{H}$. Renz contributed equally to this work.

Conflict of interest: The authors have declared that no conflict of interest exists.

Submitted: September 15, 2017 Accepted: March 29, 2018

Published: May 3, 2018

Reference information: JCI Insight. 2018;3(9):e97503. https:// doi.org/10.1172/jci.insight.97503.

\section{Introduction}

Asthma is a major chronic inflammatory disease of the airways with increasing prevalence and incidence worldwide (1). Recent clinical findings indicate a complex and heterogeneous pathophysiology among patients with asthma (2). Currently, subgroups of patients are defined on the basis of cellular and molecular signatures, which are termed endotypes $(3,4)$. Among these, the type- 2 endotype is highly prevalent, comprising about half of asthmatics, and is also well defined by the central role of T helper 2 (Th2) immunity. Th2 cells differentiate from naive $\mathrm{CD} 4^{+} \mathrm{T}$ cells, which serve as a common precursor for the functionally diverse Th cell subpopulations (5-9). In naive T cells, IL-4 induces the expression of the transcription factor GATA3, which promotes the expression of IL-4, IL-5, and IL-13. In accessory cells, these cytokines induce a variety of downstream responses resulting in tissue remodeling and severe impairment of lung function $(10,11)$.

The Th2 immune response is amplified in self-propagating loops, stabilizes over time, and escapes immune regulation by, e.g., Treg cells (12). The host remains incapable of restoring immune homeostasis. The molecular mechanisms underlying the maintenance of the allergic response in the inflamed tissue are not well defined. Hence, deciphering these signals is pivotal for understanding the pathogenic mechanisms in diseases. Recently, posttranscriptional mechanisms, including microRNAs (miRs), have been shown to control gene expression and recognized to contribute significantly to human disease (13-15). 
A

$\mathrm{ST} 2+\mathrm{Th} 2$ cells are retained in the inflamed lung

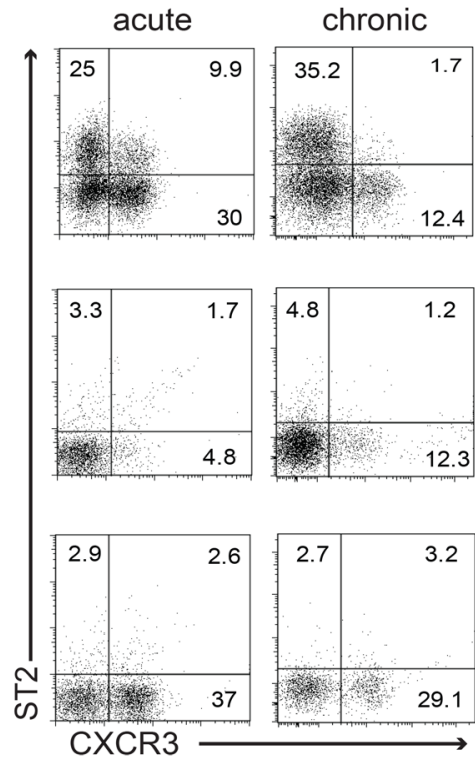

B Th2 subset stabilizes in chronic inflammation
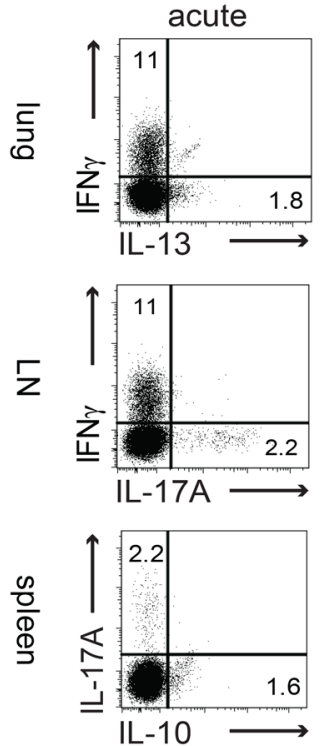
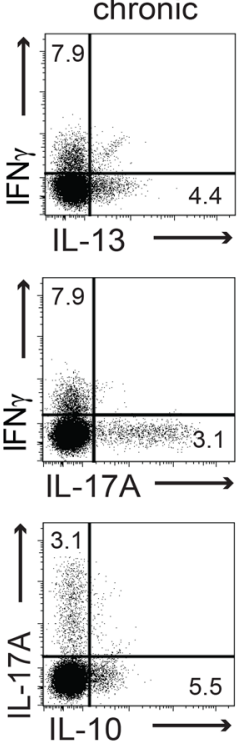

C Th- subsets

(total)

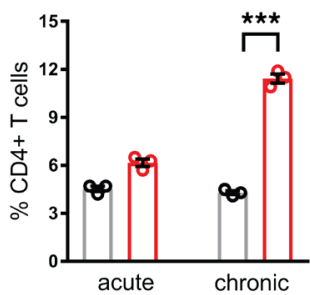

D Th- subsets (tissue resident)

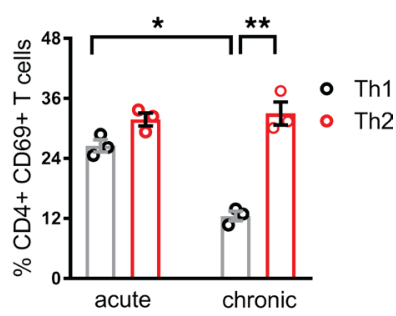

E tissue-resdident cells in the chronic inflamed lung are memory T cells

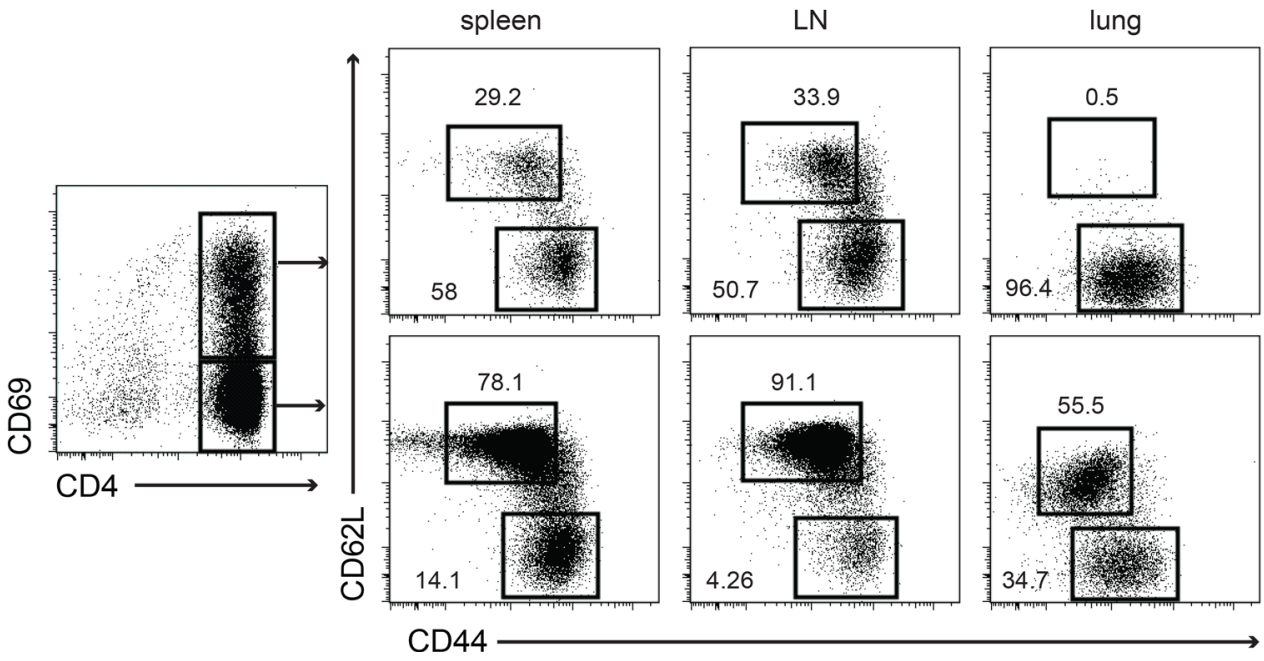

Figure 1. CD4+ST2+ Th2 cells accumulate in allergic inflamed lung tissue. (A) Flow cytometric analysis of CD4+ $T$ cells isolated from spleen, lymph node (LN), and inflamed lung tissue for the presence of CXCR3 ${ }^{+}$Th1 and ST2 ${ }^{+}$Th2 cells in the acute and chronic phase. Data are shown as percentage CD4 ${ }^{+} \mathrm{T}$ cells and are representative of 3 independent experiments. For the analysis, $n=3-4$ mice were pooled per experiment. (B) Flow cytometric analysis of intracellular cytokine production in $\mathrm{CD}^{+} \mathrm{T}$ cells isolated from acute and chronic inflamed lungs. The frequencies of (C) total Th2 and (D) tissue-resident Th2 cells were determined in OVAinduced acute and chronic allergic inflamed lung tissues using flow cytometry. (E) Tissue-resident CD4+ $T$ cells isolated from spleen, LN, and lung were stained

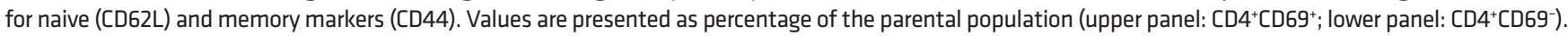
Data are representative of 2 independent experiments with $n=3-4$ mice per experiment. Graphs (C and $\mathbf{D})$ depict mean \pm SEM of 3 independent experiments each with 3-4 animals per group. ${ }^{*} P<0.05$, ${ }^{* *} P<0.01$, ${ }^{* *} P<0.001$, by (C) 2-tailed Mann-Whitney $U$ test and (D) ANOVA followed by Tukey's post test.

miRs fine-tune gene expression by facilitating mRNA degradation and repression of protein synthesis (16, 17), and an altered expression of miRs has been documented for $\mathrm{T}$ cell-driven diseases including asthma (18-22). Since miRs are encoded in genes and gene expression is strongly influenced by cytokine input in Th cells, it is likely that miR expression levels are influenced by various stimuli present in the inflamed tissue and changing micromilieus in different disease stages. Whether the miR expression pattern in $\mathrm{Th} 2$ cells is subject to further changes in chronic allergic inflammation and if these changes affect maintenance of the Th2 subset in the inflamed tissue have not been investigated thus far. 
A Lineage- specific gene expression in sorted Th- subsets: mRNA

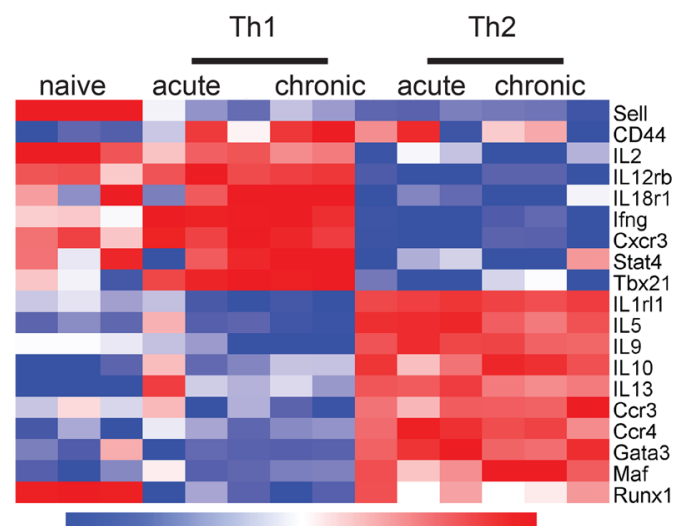

low

high

C Alignment of upregulated genes in Th2 chronic with published Th2- profiles

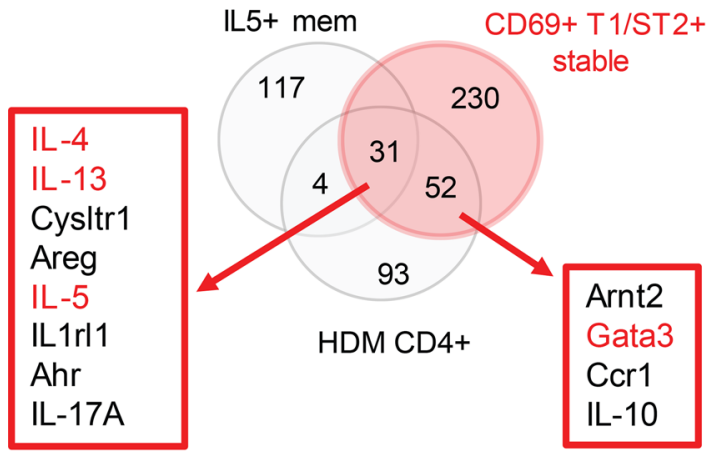

B Post-sorted validation of cytokine production in Th- subsets: protein
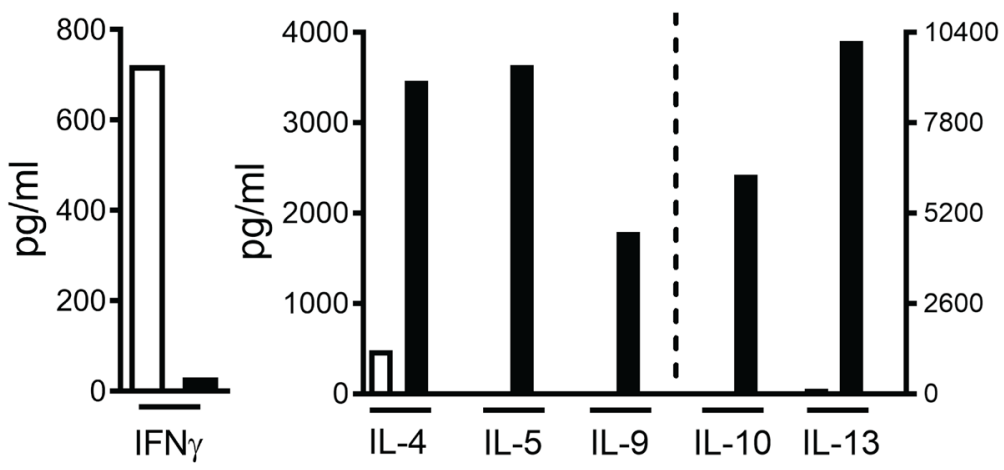

$\stackrel{8}{3}$

D

Differential gene expression naive $T$ cells vs early Th2 cells

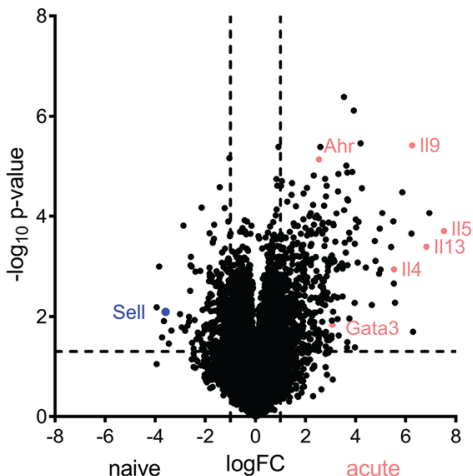

E Limited changes in gene expression over disease progression

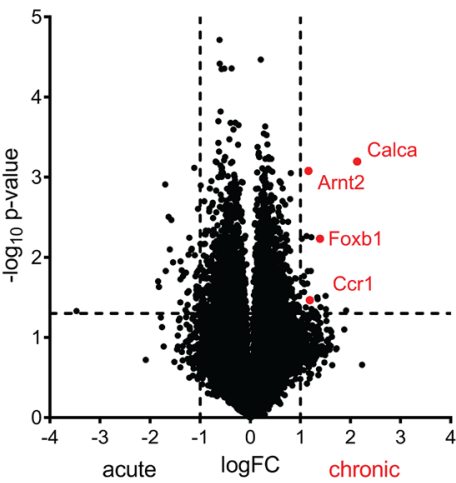

changes in transcription factor expression

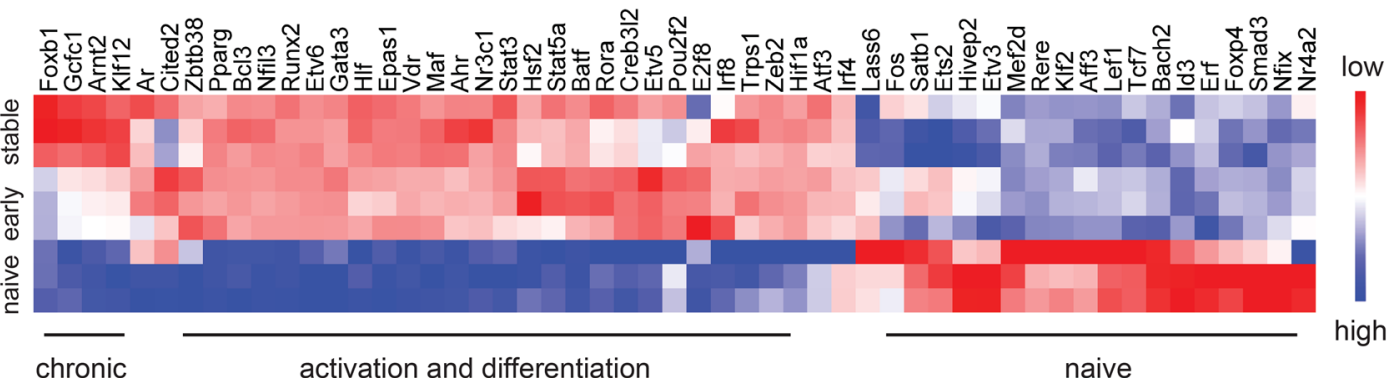

Figure 2. Isolation of live CD4 ${ }^{+}$Th cell subsets from allergic inflamed lungs using FACS. (A) Heatmap representation of expression of naive T cell and Th-subset-specific genes. (B) Cytokine expression of FACS-isolated $2 \times 10^{5}$ Th1 and Th2 cells. (C) Venn diagram comparing upregulated genes detected in memory Th2 cells with previously published lung Th2 memory data sets. IL-5+ memory (mem) (D011.10 cells polarized to Th2 in vitro and expanded in vivo, GSE33516 data set), CD4+ T cells from house dust mite-induced (HDM-induced) allergic airway inflammation (CSE72005 data set). Changes in gene expression were determined by comparing to the respective control population of each experimental setting with a cutoff of 2 -fold. (D) Volcano plot representation of gene expression differences between naive CD4+ $T$ cells and early Th2 cells. Lineage-specific genes are highlighted in color. Dashed lines indicate cutoff levels for gene expression (log2-fold $>1$ ) and significance $(P<0.05)$. (E) Volcano plot representation of gene expression changes from early to stable Th2 (memory) cells. (F) Heatmap depicting differentially expressed transcription factors. $n=3$ independent experiments. Cells were pooled from 10-12 mice. ( $\mathbf{D}$ and $\mathbf{E}$ ) Fold change in expression was calculated with mean values for each group. 
differentially expressed miRNAs in Th2 cells

$\mathbf{A}$

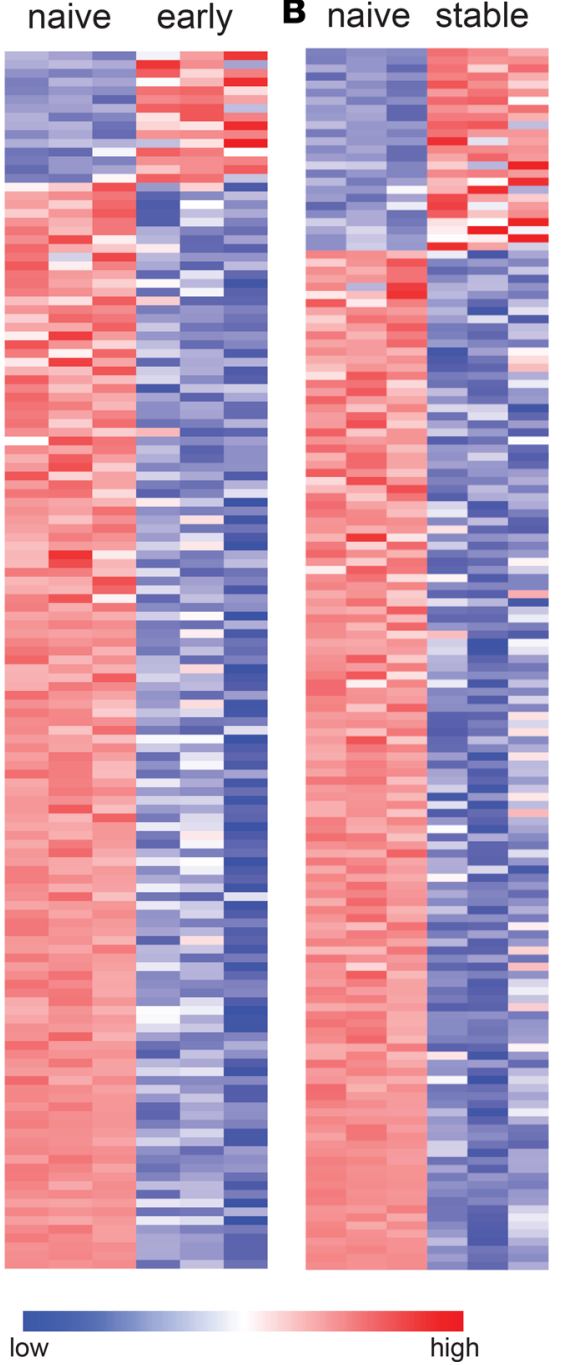

upregulated miRNAs in

C early and stable Th2 cells

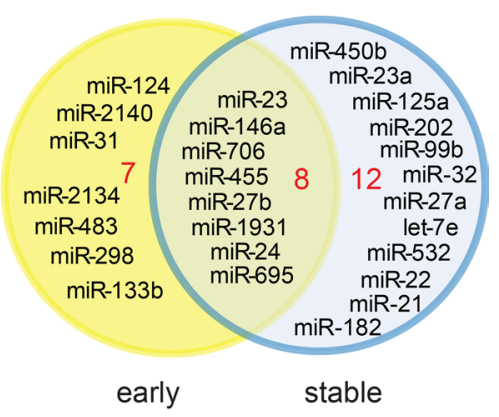

Figure 3. miR silencing in differentiated Th subsets. Heatmap depicting differentially expressed miRs in (A) early Th2 and (B) stable Th2 cells compared with naive $T$ cells. (C) Venn diagram summarizing upregulated miRs in early and stable Th2 cells.

The broad target spectrum of a single miR makes it is difficult to predict the consequence of miR regulation on cellular processes unless miR-regulated gene and protein expression are considered. Systems biology approaches successfully map such interactome and miRNome data for the identification of regulatory pathways in disease (23-30).

Here we developed a network-based framework, the Impact of Differential Expression Across Layers (IDEAL), that prioritizes miRs based on their interaction patterns in the local neighborhood of differentially expressed (DE) genes (disease module). To measure the cumulative influence of miRs in the miR-mRNA and mRNA-mRNA multilayer network, we introduced collective impact (CI) as a measure. This approach identified a subset of 5 asthma-related miRs that have maximal topological impact. Interestingly, these miRs display only moderate fold change (FC) and would not be captured by traditional network-agnostic methods. Finally, we validate these findings in vitro by antagonizing their activity in $\mathrm{Th} 2$ cells and detected lower production of the Th2-specific effector cytokines IL-5, IL-9, and IL-13.

\section{Results}

$C D 4^{+}$Th2 cell population stabilizes in chronic inflamed airways. To delineate the contribution of miRs in stabilizing the asthmatic phenotype at the level of Th2 cells, an established mouse model of allergic asthma was employed. The allergen-induced (we used ovalbumin [OVA]) phenotype has an acute inflammatory component that progresses into the chronic phase marked by airway remodeling (Supplemental Figure 1 ; supplemental material available online with this article; https://doi.org/10.1172/jci.insight.97503DS1). 
A workflow

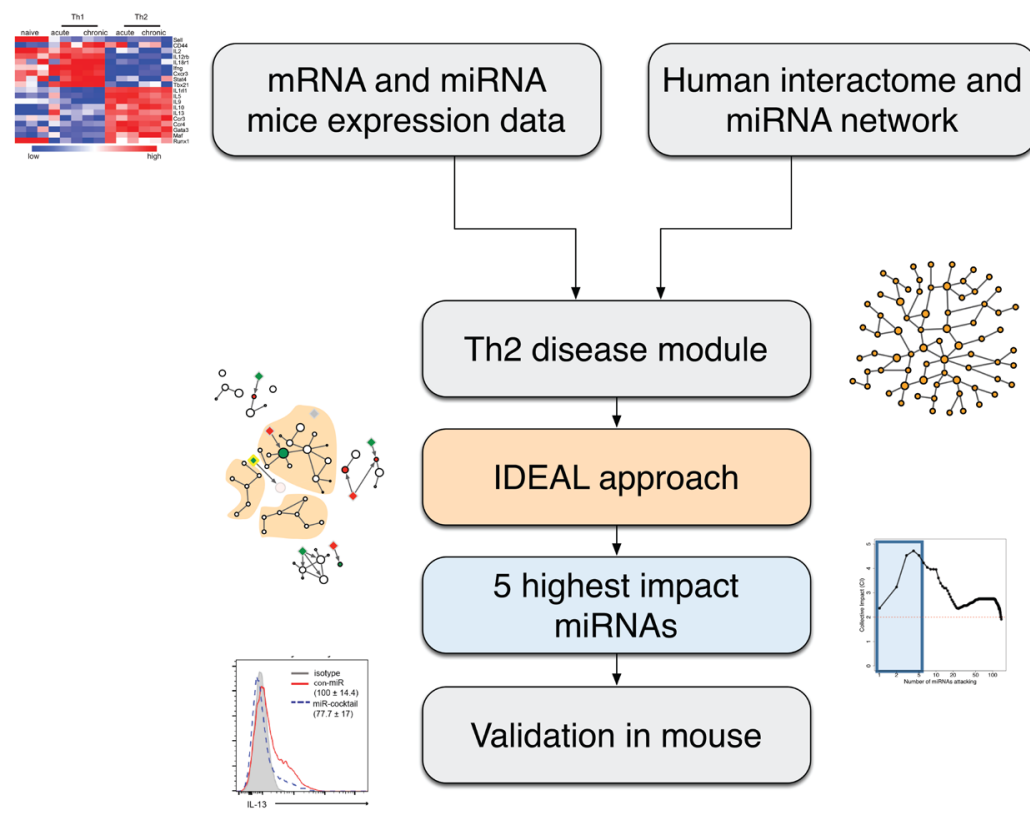

miRNAs impact on

building the Th2- modules
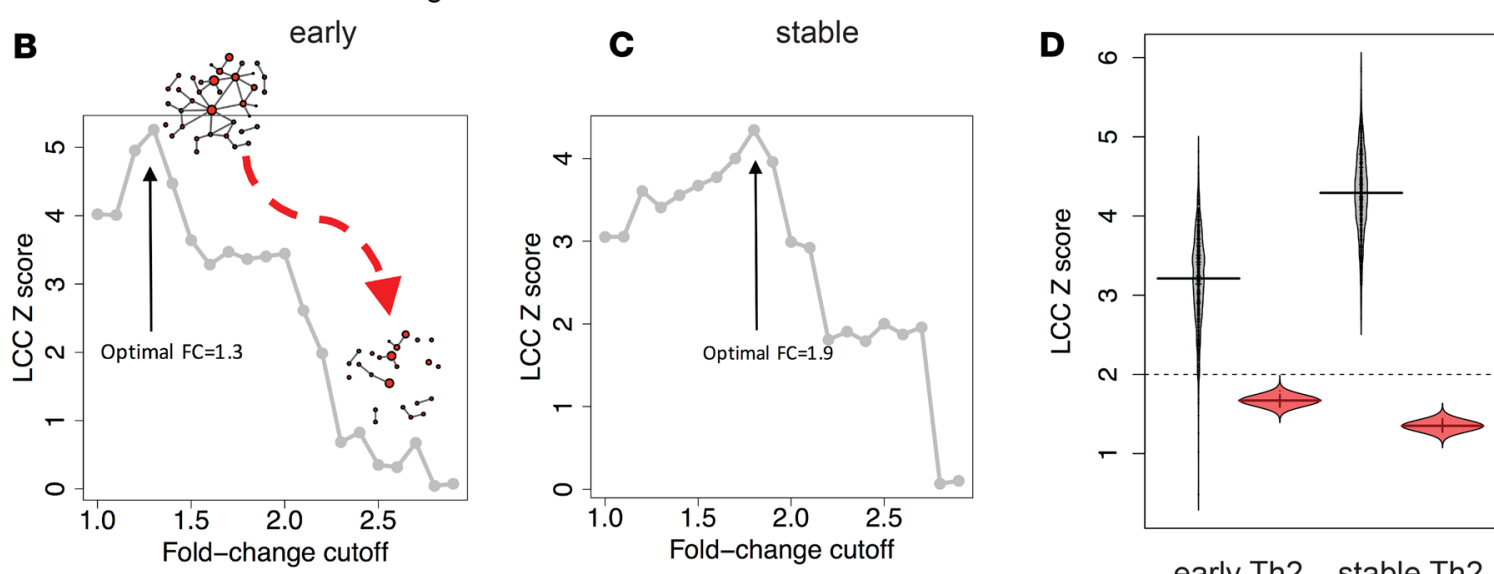

$\square$ random

$\square$ observed

early Th2 stable Th2

Figure 4. Th2 differentially expressed genes form disease modules in the miR-interactome network. (A) Workflow of the methodology used to prioritize miRs relevant to the Th2 phenotype. Expression data were collected and differentially expressed genes and miRs were mapped to the human miR-interactome, resulting in a stable and an early disease module. We developed the Impact of Differential Expression Across Layers (IDEAL) methodology to prioritize miRs that target topologically central genes in the respective disease modules. A collective of 5 miRs was found to have maximum impact in the stable Th2 condition. These miRs were ultimately validated in mice. (B) Genes differentially expressed in stable Th2 cells above a certain fold-change (FC) cutoff are mapped onto the interactome. The size of the obtained largest connected component (LCC) is compared to that of a random set of genes of the same size, resulting in a $Z$ score. This procedure is repeated for multiple cutoffs. A maximum connectivity is observed for $F C=1.3$. (C) Same as B, for the early condition. An optimal cutoff of 1.9 is found. (D) The impact of differentially expressed miRs of the Th2 disease modules is assessed by removing their targets having opposite FC. The $Z$ score of the resulting modules are shown by red beans and are nonsignificant (below the red dashed line corresponding to $95 \% \mathrm{CI}$ ). However, the modules show high robustness to the removal of the same number of randomly picked nodes, simulating random attacks, with $Z$ scores consistently significant (gray beans).

We observed that transition into the chronic stage was accompanied by changes in Th-subset recruitment to the inflamed lung (Figure 1A). Supporting the hypothesis that the pathophysiology of this model is driven by Th2 cells, $\mathrm{CD}^{+} \mathrm{IL}_{-1}-13^{+}$and $\mathrm{ST} 2^{+} \mathrm{Th} 2$ cell numbers (hereafter named Th2) increased from the acute to the chronic stage (Figure 1, A-D) and accounted for about one third of the activated/resident CD4 ${ }^{+}$ 


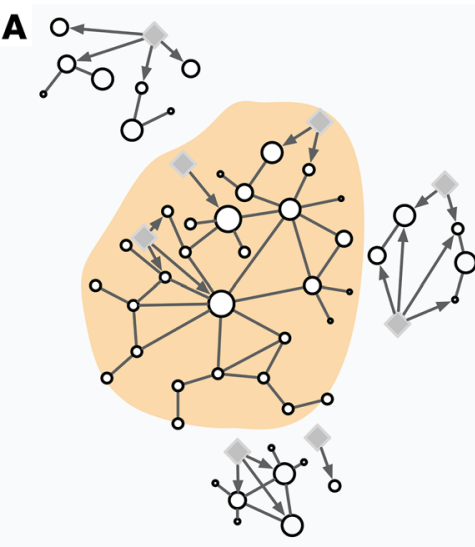

Th2 disease module in the miRNA-mRNA interactome

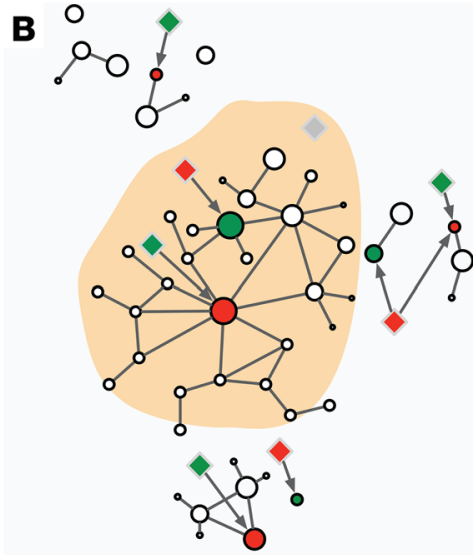

Differentially expressed miRNAmRNA in opposite direction

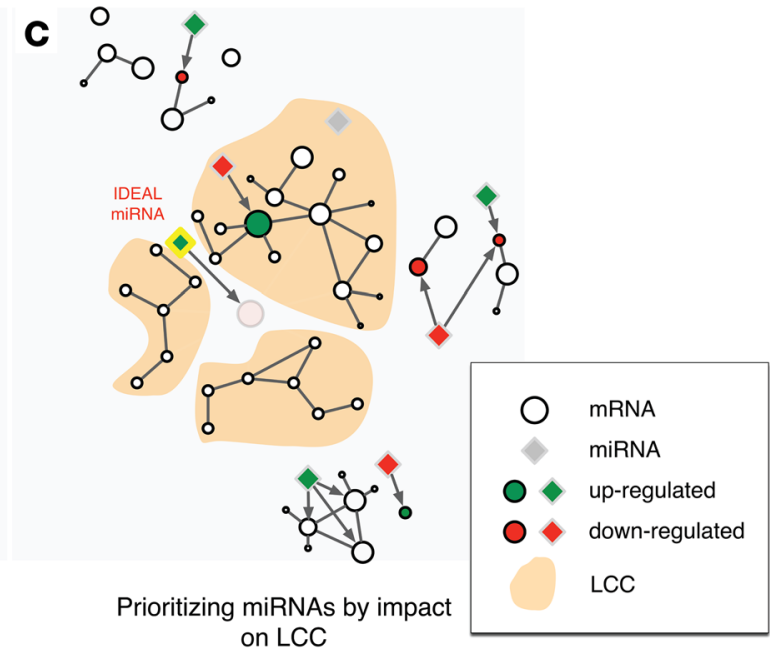
on LCC

Figure 5. Prioritizing miRs with maximum collective impact. To prioritize important differentially expressed (DE) miRs, we developed a method for measuring their collective topological impact on a disease module, the Impact of Differential Expression Across Layers (IDEAL). (A) IDEAL starts with a disease module formed by the disease genes (circles) in the interactome, with a set of DE miRs (diamonds) targeting it. The largest connected component (LCC) is shown in orange. (B) Interactions between miRs and mRNAs of opposite fold change (FC) are retained. (C) The central miR has maximal impact, measured by the decrease in LCC size when removing its target, and is therefore the first IDEAL miR. The procedure then iterates to find the new miR with most impact, removes its targets, and proceeds until all miRs have been ranked.

$\mathrm{T}$ cell population in the lung tissue (Figure 1, A and D), as compared with other immunological organs such as the spleen or lymph node (Figure $1 \mathrm{~A}$ ). In lungs, small but rising IL- $17 \mathrm{~A}^{+}$and $\mathrm{IL}-10^{+} \mathrm{CD} 4^{+} \mathrm{T}$ cell populations were detected as well (Figure 1B), indicating a coevolution of Th17 and regulatory $\mathrm{T}$ cells next to Th2 cells. In contrast, lung-tissue-resident $\mathrm{CD} 4^{+} \mathrm{CD} 69^{+} \mathrm{CXCR} 3^{+} \mathrm{Th} 1$ cells (hereafter referred to as Th1) decreased over time (Figure 1, A and D). In chronic inflamed airways, tissue-resident cells showed a memory phenotype as analyzed by $\mathrm{CD} 4{ }^{+} \mathrm{CD} 44^{+} \mathrm{CD} 62 \mathrm{~L}^{-}$(Figure $1 \mathrm{E}$ ). Thus, the established Th2 population stabilizes over time and is actively retained in inflamed airways.

Isolation and profiling of tissue-resident Th2 cells. Tissue-resident Th1 and Th2 cell subsets were purified from inflamed lungs by cell sorting (Supplemental Figure 2) and validated based on their cytokine production and gene expression profile. For gene expression studies, naive $\mathrm{CD} 4^{+} \mathrm{CD} 62 \mathrm{~L}^{+} \mathrm{CD} 44^{+} \mathrm{T}$ cells isolated from spleen were used as control population (Figure 2B). Comparing the expression levels of lineage- and subset-specific transcription factors, surface markers, and cytokine genes confirmed the purity and phenotype of the sorted subsets (Figure 2A). Th2 cells produced high amounts of the signature cytokines IL-4, IL-5, IL-9, IL-10, and IL-13, while Th1 cells secreted high amounts of IFN- $\gamma$ (Figure 2B). Relating the Th2 gene expression profile to previously published data from Th2-driven models $(31,32)$ confirmed an excellent coverage of a core set of Th2-specific genes (Figure 2C). In early Th2 cells (isolated from acute inflamed lungs), 669 genes were DE compared with naive T cells, and 695 in stable Th2 cells (chronic inflamed lungs and memory phenotype). Among the most strongly upregulated genes are the Th2-specfic cytokines IL-5, IL-9, IL-13, as well as GATA-3 (Figure 2D). Genes upregulated in early and stable Th2 cells versus naive T cells are compiled in Supplemental Tables 1 and 3. Genes downregulated in early and stable Th2 cells versus naive T cells are compiled in Supplemental Tables 2 and 4. Pathway enrichment of upregulated genes retrieved asthma- and inflammatory bowel disease-related pathways (Supplemental Tables 5 and 6). Comparison of early and stable Th2 cells identified $38 \mathrm{DE}$ genes. The set of downregulated genes in stable Th2 cells mostly comprised genes coding for histone family members. In stable Th2 cells, the transcription factors Arnt2, Foxb1, Ccr1, and Calca (Figure 2, E and F) were upregulated. Calca has previously been described to play a role in dendritic cell priming in asthma $(33,34)$.

Our analysis uncovered a markedly altered miR expression pattern. Compared with naive $\mathrm{T}$ cells, both early (Figure 3A) and stable (Figure 3B) Th2 cell subsets showed a massive reduction of miR expression, suggesting a loss of regulation of miRs during differentiation (35). Although approximately $90 \%$ of 
A
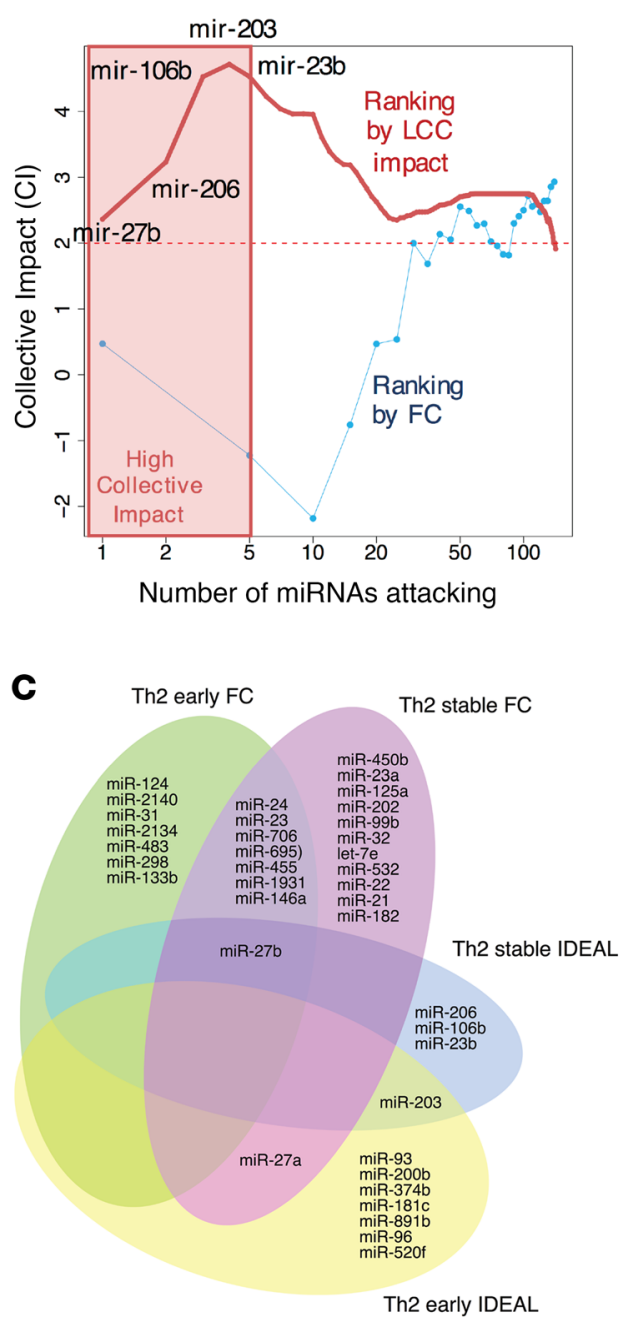

B early Th2

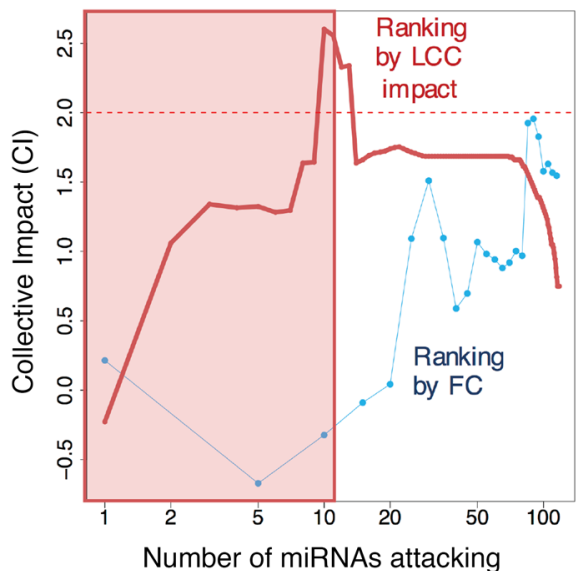

D

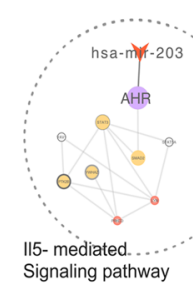
Signaling pathway

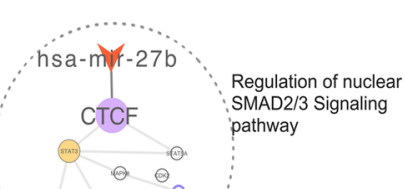

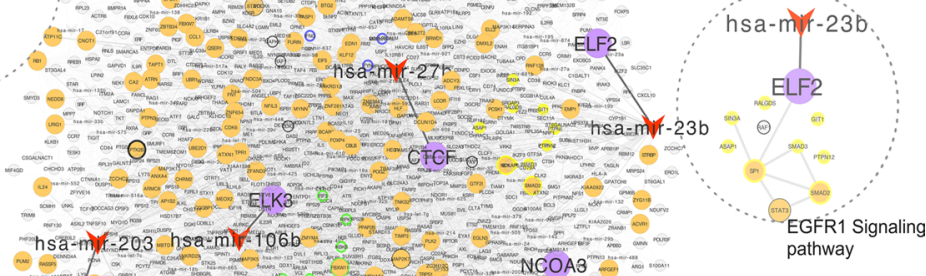

$(x)$
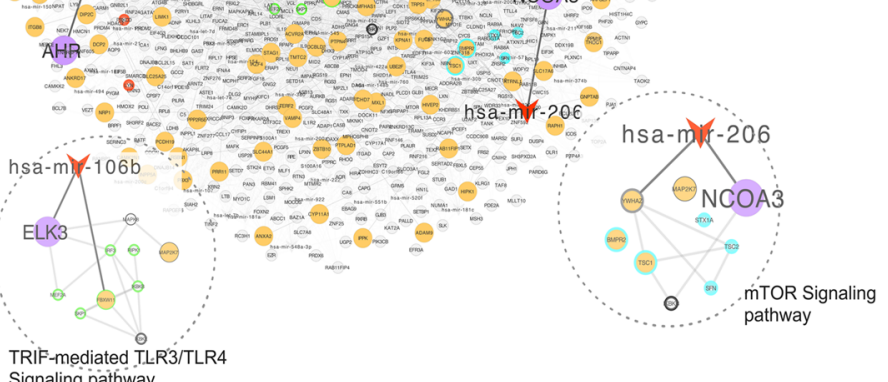

Signaling pathway

Figure 6. IDEAL predicts the collective impact of miRs in stable Th2 cells. (A) The cumulative impact after removing $n$ miRs is measured by the collective impact (Cl), a measure that systematically compares the largest connected component (LCC) relative size after attack by $n$ miRs to that expected at random. A peak of $\mathrm{Cl}$ at approximately $5 \mathrm{miRs}$ was observed, indicating maximal impact for stable Th2 and approximately 10 miRs for early Th2 (B). (C) Venn diagram comparing the top-ranking miRs with fold-change (FC) and Impact of Differential Expression Across Layers (IDEAL) methods. (D) The stable Th2 disease module is shown (genes, circles; miRs, V's). The top 5 miRs are highlighted in red, and their targets in dark red. Node size is proportional to degree. The subnetwork highlights the top pathways enriched among the target genes and first neighbors of particular $\mathrm{miR}$ in the interactome. The blue circles mark the mTOR, yellow the EGFR1, purple the TGF/SMAD, red the IL-5, and green the TRIF pathway. The common nodes among the pathways are circled in black.

naive $\mathrm{T}$ cell miRs disappear, a small specific set of 15 miRs remained upregulated in early Th2 cells and 20 in stable Th2 cells. Eight out of these 35 miRs were expressed in both subsets (Figure 3C).

Impact of miRs on the Th2 disease modules. Given that each miR usually regulates multiple mRNA targets, it is crucial to integrate the connectivity structure of miR and mRNA networks to understand the dynamics of the Th2 signature at the molecular level. Recent advances in miRNome and interactome mapping demonstrate the maturity and value of such systems biology approaches in discovering regulatory pathways in diseases (23-30). Moving a step forward, integrating the miRNome and interactome along with the transcriptome expression allowed us to prioritize miRs with significant collective impact (Figure 4A). We used 
Table 1. Top 10 pathways formed by IDEAL miRs in the molecular interactome

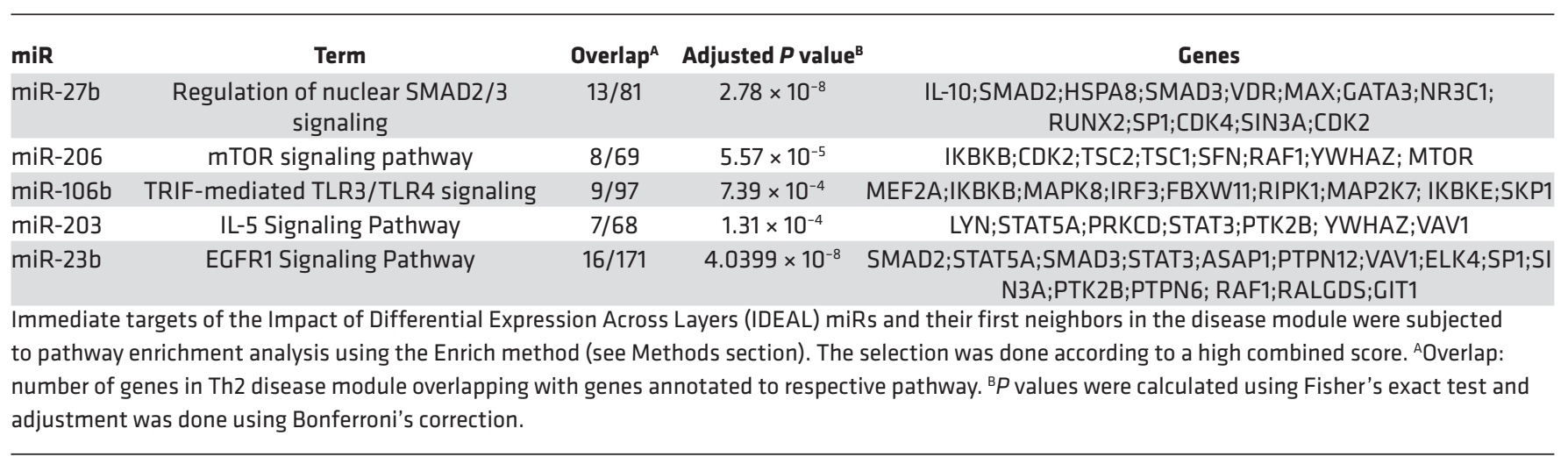

the human protein-protein-interaction (PPI) and miR-mRNA networks, with the established observation that human PPI networks are well conserved in mouse $(36,37)$ (see Methods).

Disease genes tend to aggregate in localized neighborhoods of the interactome defined as disease modules (29). Here we investigated if the DE genes with respect to naive Th cells followed the same clustering pattern. DE genes above various FC cutoffs were mapped to the human interactome, and the aggregation of the corresponding proteins was measured by the size of the largest connected component (LCC) - the number of nodes in the largest connected subgraph. Comparison to random sets of genes of the same size allowed us to derive a significance $Z$ score (see Methods). We observed that $\mathrm{DE}$ genes form highly interconnected clusters for intermediate FCs ( $\mathrm{FC}=1.3, Z=5.3$ for stable Th2, Figure 4B; and FC = 1.8, $Z=4.3$ for early Th2, Figure 4C). These FCs were chosen to define the early and stable Th2 disease modules. This methodology relies only on connectivity patterns between DE genes, thereby avoiding the traditional pitfall of having to choose a predetermined FC cutoff.

Next we asked whether the DE miRs were impacting topologically central targets in the obtained disease modules. In each condition, the impact of DE miRs was evaluated by removing their predicted targets in the corresponding disease module, an approach reminiscent of the attack on scale-free networks (38). In both early and stable cases, we found that the cohesiveness of the modules was lost after target removal, with LCC sizes falling to nonsignificant levels (Figure 4D, red beans). On the other hand, the modules were still cohesive under a random attack of the same number of targets (Figure $4 \mathrm{D}$, gray beans).

Prioritization of miRs by their impact on the disease modules. Next it was examined whether a subset of DE miRs was driving the observed impact. The network model, IDEAL, captures the topological impact of several miRs in a defined neighborhood within the interactome (see Figure 5, A-C and Methods). IDEAL ranks miRs by the strength of their impact on the disease module, as measured by the decrease in LCC size when the miR targets are removed. The target genes from the top miR were excluded and removed, and the selection process was repeated iteratively. To measure the impact of this successive removal of miR targets, a topological measure, the CI was developed (see Methods). The CI measures the strength of the impact on LCC relative to the impact observed for random attacks. The results are shown in Figure 6A in the case of the stable Th2 condition. Among the $142 \mathrm{DE}$ miRs, the $\mathrm{CI}$ is maximized with the 5 top miRs, and then quickly decreases to a lower but still significant level of $\mathrm{CI}=2$, corresponding to the gap observed between the red and gray beans in Figure 4D. The disease module and miRs are shown in Figure 6B. Interestingly, ranking the miRs by decreasing FC had an opposite effect, where the most DE miRs showed less impact than expected at random (Figure 6A). This suggests that the most DE miRs impact peripheral nodes of the disease module, yielding an orthogonal prioritization list compared to IDEAL. A similar behavior in the case of early Th2 was observed, with $10 \mathrm{miRs}$ maximizing the collective impact for IDEAL (Figure 6B).

The miRs from IDEAL and from FC are compared in Figure 6C. FC ranking yields a substantial list of similar candidates between early and stable conditions. On the other hand, IDEAL ranking yields orthogonal sets that are highly specific to each condition, with only miR-203 being shared between early and stable conditions. Finally, miR-27b, the top ranked candidate from the IDEAL ranking in stable Th2, was also predicted to have high FC in both early and stable conditions, making it a strong candidate. 
A

Validation of miRNA induced silencing

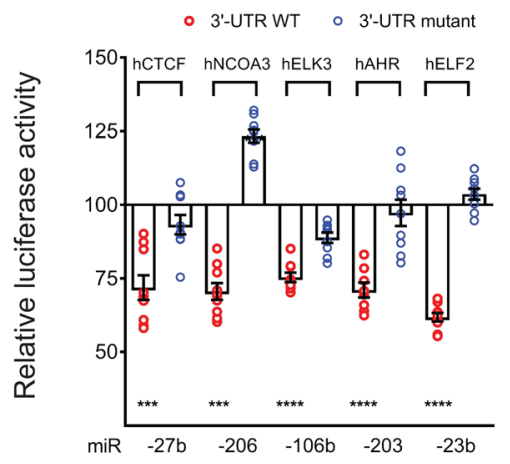

C Expression of IDEAL-targets in Th2 cells

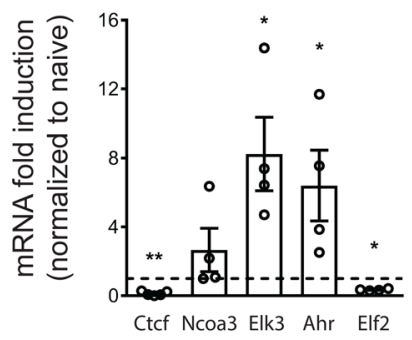

E Frequency of Th2 cell analysis flow cytometry

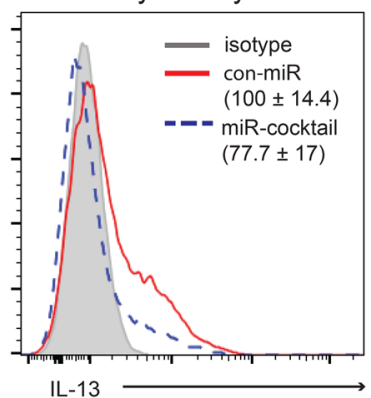

B Expression of IDEAL-miRNA in Th2 cells

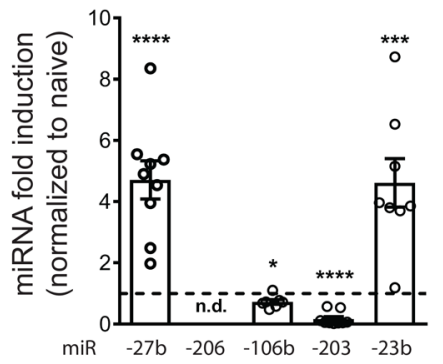

D Nucleofection of Th2 cells

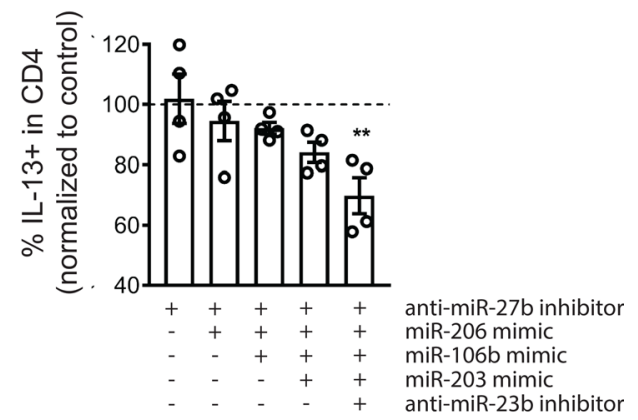

F Th2 cytokines: protein

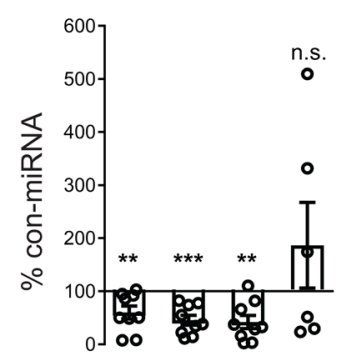

IL-5 IL-9 IL-13 IL-17
Figure 7. Targeting a miR network interferes with cytokine production in Th2 cells in vitro. (A) The WT and mutant $3^{\prime}$-UTR sequences of the human genes CTCF (target to miR-27b), NCOA3 (miR-206), ELK3 (miR-106b), AHR (miR-203), and ELF2 (miR-23b) were cotransfected with miR mimics or a scrambled sequence (con-miR). Relative light units (RLUs) were normalized to the respective control group and are presented as relative luciferase activity. Results of 3 independent experiments with $n=3$ per group are shown. (B) Relative expression of target miRs in in vitro-polarized murine Th2 cells was determined by real-time RT-PCR and normalized to naive $\mathrm{CD} 4^{+} \mathrm{T}$ cells. Graph summarizing results of 3 independent experiments with $n=2-3$ per group. (C) Relative mRNA expression, depicted as fold change, of the selected miR targets Ctcf, Ncoa3, Elk3, Ahr, and Elf2 in murine Th2 cells normalized to naive $\mathrm{CD}^{+} \mathrm{T}$ cells in $n=4-5$ samples. (D) Graph summarizing the results

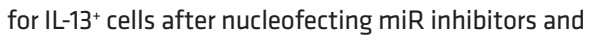
$\mathrm{miR}$ mimics as indicated in the table below the bar graph and antagonizing the IDEAL (Impact of Differential Expression Across Layers) predictions. In each condition, the con-miR transfection contained the equal amount and composition of control sequences (see Methods section). Values were normalized to the respective con-miR samples and summarize 4 independent experiments with $n=2-3$ replicates per experiment. (E) Representative histogram showing the effect of antagonizing the miR expression pattern in Th2 cells on IL-13 expression. A miR cocktail containing anti-miR-27b, miR-206 mimic, miR-106b mimic, miR-203 mimic, and anti-miR-23b was nucleofected. $\mathrm{IL}^{-13^{+}}$cells were determined by flow cytometry (blue dashed line). The effect was controlled by nucleofecting the respective amount of scrambled miR mimics/inhibitors (con-miR, red line; see Methods). Shaded histogram indicates samples stained with isotype control antibody. Values summarize 4-5 independent experiments with $n=2-3$ replicates per experiment. (F) Twenty-four hours after nucleofection, cells were stimulated with anti-CD3/ anti-CD28 for 24 hours and cytokines were measured in culture supernatant. Concentrations were normalized to con-miR sample. Graph summarizes results of 4 independent experiments with $n=2-3$ replicates per group and experiment. All graphs with quantitative data show mean \pm SEM. ${ }^{*} P<0.05,{ }^{* *} P<0.01$, ${ }^{* * *} P<0.001$ by 2 -tailed Mann-Whitney $U$ test $(\mathbf{D}-\mathbf{C})$.

Overall, we found that a small set of miRs ( 5 of $142 ; \sim 4 \%$ of all DE miRs) had a maximal CI on the stable Th2 disease module. This set comprised a combination of upregulated (miR-27b and $-23 \mathrm{~b}$ ) and silenced (miR-206, miR-106b, and miR-203) miRs. To investigate the possible biological role of these miRs, the immediate miR targets and their first neighbors in the interactome were subjected to pathway analyses (see Methods section, Table 1, and Figure 6D). The top pathway targeted by miR-27b was nuclear SMAD2/3, and the EGFR1 signaling pathway is regulated by miR-23. Both pathways are associated with immunosuppression by, and activity of, regulatory $\mathrm{T}$ cells $(39,40)$. Active silencing of these pathways in stable Th2 cells may help to escape intrinsic immune regulation. In contrast, miRs silenced in Th2 cells actively target components of signaling pathways, which are associated with polarization (41), activation and proliferation (42), as well as act downstream of cytokines $(43,44)$, which are exemplified for IL-5. Taken together, these results show that suppressing the activity of these specific miRs in combination with active silencing of immunosuppressive pathways might confer Th2 cell maintenance. 
Targeting IDEAL miRs alleviates cytokine production in Th2 cells. This predicted set of miRs was further validated in several functional assays. For each $\mathrm{miR}$, a target gene with both strong connectivity within the disease module and human-to-mouse seed sequence conservation was selected for further analysis (Supplemental Figure 3, Methods). This resulted in the selection of CTCF for miR-27b, NCOA3 for miR-206, ELK3 for miR-106b, AHR for miR-203, and ELF2 for miR-23b. A dual reporter luciferase assay revealed regulation of the selected target genes by the respective miRs, as shown by the marked and significant reduction of the luciferase signal detected for all wild-type (WT) 3'-UTR sequences. Sequence specificity was validated by deleting the miR seed sequences (mutant), resulting in an unaltered luciferase signal (Figure 7A). These results confirm regulation of genes, central to multiple pathways merging into inflammation, by IDEAL miRs.

Next, it was examined if the miRs are essential for the stability of the Th2 phenotype. For this purpose, miR expression was analyzed in primary in vitro-generated $\mathrm{Th} 2$ cells and compared to naive $\mathrm{CD} 4^{+} \mathrm{T}$ cells. Upregulation of miR-27b and $-23 b$ was confirmed together with a relative suppression of miR-106b and -203 . The only exception was miR-206, which is considered to be muscle specific (45) and remained undetectable by standard TaqMan RT-PCR in both naive $\mathrm{CD}^{+} \mathrm{T}$ cells and Th2 cells (Figure 7B). The same samples and comparison was used to confirm the expression levels of the selected miR target genes (Figure 7C). To assess if this unique rearrangement of the miRNome upon differentiation is required for Th2-specific function, respective miR functions were antagonized. Upregulated miRs were blocked by $\mathrm{miR}$ inhibitors (anti-miR-27b and anti-miR-23b) and miRs, silenced during the polarization process, were overexpressed by miR mimics (miR mimics for miR-206, miR-106b, and miR-203). To reproduce the CI (Figure 3D) experimentally, the IDEAL miRs were antagonized in the predicted ranking and order by nucleofection. Approaching the prediction of a collective impact of miRs, a progressive reduction of the Th2 phenotype was detected resulting in a significant reduction of IL-13 in Th2 cells, with the simultaneous antagonization of all 5 predicted miRs (Figure 7, D and E). In each condition, an equal dose of control mimics and antagonists (miR Mimic Negative Control \#1 and Anti-miR miR inhibitor Negative Control \#1, both from Ambion) was added, and viability of the cells after nucleofection was confirmed as well. The collective impact of this approach resulted in marked reduction of IL-5 (40\% compared with con-miR), IL-9 (54.5\%), and IL-13 (58\%) production in Th2 cells. Conversely, IL-17A production, which is not associated with $\mathrm{Th} 2$ cell function, was induced (Figure $7 \mathrm{~F}$ ). These data indicate that at least a substantial portion of the Th2 phenotype depends on the combined impact of a predefined set of miR activation.

\section{Discussion}

Allergic airway inflammation is repetitively initiated upon allergen contact and is accompanied by asthma symptoms causing distress in patients. Thus far, it is unclear how the Th2 immune response surpasses phenomena like exhaustion of the immune response or tolerance induction by Treg cells. This study describes the development of a network-based framework to systematically explore the impact of miRs in maintaining $\mathrm{CD}^{+} \mathrm{Th} 2$ cells in inflammation. miRs control a variety of signaling pathways and cellular processes in health and disease. However, in the majority of cases, miR-mediated mRNA depletion is mild (46). The ability of a single miR to target several hundred genes enables these molecules to effectively fine-tune many cellular events simultaneously. If these targets are enriched for genes participating in common signaling pathways, the sum of single modest interactions is expected to yield a stronger response. Additionally, single mRNAs can harbor more than one complementary binding site for miRs and predicting the net result of miR activity in such a setting remains challenging. There is growing consensus that instead of analyzing single miR-single gene interactions, miR function is best understood when mapped in a network context (47). We used an approach that integrates miRNome and proteome interactions along with the transcriptome expression results. Our goal was to identify miRs that target genes with atypically high connections within the interactome (hubs) and therefore might evoke a strong effect (48).

This systems immunology approach — IDEAL — identifies key gene-regulatory alterations evoked by miRs in the whole set of molecular interactions in a cell (interactome). IDEAL further weighs the net consequences of miR activity on the interactome by applying a network-based prioritization algorithm. Assessing the cumulative perturbation outcome to determine the number and identity of miRs, which together cross the threshold of quiescence and lead to inflammation, IDEAL predicted a maximal collective impact of 5 miRs in stable Th2 cells, comprising up- and downregulated miRs. Antagonizing the expression level of these miRs in vitro significantly reduced cytokine production in Th2 cells. Overall, we believe this is the first study describing changes in $\mathrm{miR}$ expression as a mechanism in driving persistence of the allergic immune response. 
To assess the impact of miRs on stable Th2 cells, lung-tissue-resident Th2 cells with a memory phenotype $\left(\mathrm{CD} 4^{+} \mathrm{CD} 69^{+} \mathrm{CD} 44^{+} \mathrm{CD} 62 \mathrm{~L}^{-}\right)$were analyzed. Since $\mathrm{Th} 2$ polarization is initiated in naive $\mathrm{CD}^{+} \mathrm{T}$ cells, these cells were chosen as the reference population to address the question of Th2 cell maintenance in chronic inflamed airways. We did not investigate differences in miR expression between polarized Th subsets, e.g., comparing Th2 cells with Th1 cells, since transcriptional programs activated in early Th2 cells further stabilize throughout disease progression. Changes occurring within the Th2 subset are more likely to explain intrinsic mechanisms ensuring maintenance of these cells in chronic inflammation. Furthermore, activated transcriptional programs differ significantly between distinct Th subsets and it is likely that the events observed in Th2 cells evolve independently from events observed in other Th subsets. However, we cannot exclude similar mechanisms in other Th subsets as well. We observed the upregulation of only a few and downregulation of many miRs in polarized Th2 cells compared with naive T cells. Silencing of miR expression in $\mathrm{T}$ cells has been documented previously and shown to occur downstream of $\mathrm{T}$ cell receptor engagement (35). Considering the important role of miRs in maintaining immune homeostasis (14), it appears reasonable that for the induction of specialized transcriptional programs during effector subset polarization, inhibitory miRs need to be silenced. Therefore, loss of miR regulation should be taken into account for disease development as well. IDEAL considered the absolute FC levels of miRs to identify relevant miR-gene interactions. To estimate the impact of these alterations on the molecular interactome, IDEAL considered the consequences resulting from perturbing the downstream interconnections of targeted genes, rather than the FC expression of miRs. IDEAL proposes miRs with a modest change in expression, which target genes with atypically high numbers of connections. Since a singular miR effect is considered to be rather mild, we introduced the measure CI and assessed the cumulative perturbation outcome to identify the number and identity of miRs, which together cross the threshold of quiescence and lead to inflammation.

To control IDEAL predictions, we simulated the CI for miRs with the highest FC as well. Our model proposes a stabilizing effect of the first $10 \mathrm{miRs}$, as shown by the negative CI value. These miRs are downregulated compared with naive $\mathrm{T}$ cells and this list comprises, e.g., miR-181a (FC: -220$)$, miR-181b (FC: -53), miR-422 (FC: -27), miR-30e (FC: -25), and miR-378 (FC: -21). The miR-181 family has been reported to target genes important for $\mathrm{T}$ cell function and the capability to be retained at the inflamed site, including TCR, CD69, as well as the anti-apoptotic Bcl-2 (49). Suppressing this miR family in memory Th subsets might therefore ensure survival. The role of miR-422 has been been investigated in tumor biology, where its antiproliferative activity was described (50). miR-30e and miR-378 have not been addressed in Th2 cells thus far. In NK cells, both are actively silenced after activation in humans and mice, and have been shown to target granzyme and perforin genes. Both gene products determine the cytotoxicity of NK cells (51). Since the same miR downregulation is observed in Th1 cells as well (A. K1lıç, unpublished observations), we conclude that silencing of these miRs is a general mechanism that stabilizes and ensures proper immune response by $\mathrm{CD} 4^{+} \mathrm{T}$ cells rather than controlling Th2-specific functions.

An important role for deregulated expression of $\mathrm{miR}$ in disease has been established for a variety of conditions, including asthma. Studies performed in humans and mice have investigated different serological and structural compartments and identified DE miRs. In airway smooth muscle cells of severe asthmatics, the expression of miR-221 is elevated compared with nonsevere asthma and controls and shown to regulate hyperproliferation of airway smooth muscle (52). For airway epithelia from asthmatics, higher expression of miR-21 and miR-126 as direct IL-13 targets was reported. miR-21 was shown to drive steroid-insensitive asthma (22), while miR-126 is associated with Th2 inflammation (53). The latter has been proposed by the authors as an important miR in the initiation phase of allergic inflammation. In mice, blockade of either miR was shown to alleviate the allergic phenotype in house dust mite (HDM) and OVA models of allergic airway inflammation. Studies in miR-155-KO mice provided evidence for reduced asthma symptoms in the absence of miR-155 (54). Yet, targeting strategies with miR inhibitors in the WT situation failed to alleviate the asthmatic phenotype (55). Among these presented miRs, our data support Th2 specificity of miR126 , which has previously been detected in allergic inflamed airway tissue and which we find elevated in tissue-resident early Th2 cells, but normalized in the chronic situation in stable Th2 cells. In a recent study, elevated miR-19a was described in airway-infiltrating $\mathrm{CD}^{+} \mathrm{T}$ cells of asthmatic patients compared with $\mathrm{CD}^{+} \mathrm{T}$ cells isolated from healthy controls (20). miR-19a belongs to the miR-17-92 cluster family, which we find downregulated in early and stable Th2 cells compared with naive CD4 ${ }^{+} \mathrm{T}$ cells. However, since the $\mathrm{CD}^{+} \mathrm{T}$ cell infiltrate in asthmatic airways in humans and mice is far more complex than only Th2 cells, it is possible that other Th subsets contribute to the elevated miR-19a expression. 
Instead of predicting a single miR with a detrimental effect, IDEAL identified 5 miRs, which act collectively to evoke the maximal impact. Against our expectation, the identified miRs comprised both induced and silenced miRs, implicating that next to active RNA interference, initially silenced pathways and processes must be admitted in polarized Th cells. The miR-23-24-27-family members miR-27b and miR-23b are induced in stable Th2 cells. Initial reports in miR-transgenic and -KO mice identified this cluster as Th2-suppressing miRs $(21,56)$. More recent studies provide evidence that especially elevated levels of miR-27b perturbs Treg cell function and therefore blocks tolerance induction (57). We performed pathway analyses for the miR-27b and miR-23b targets and their first neighbors in the interactome and found immune-regulatory pathways, like TGF/SMAD and EGFR1, among the top hits. Targeting these pathways might provide mechanisms for escaping endogenous immune regulation. Next to immune escape, immune-stimulating pathways promoting survival and proliferation need to be established as well. As such, pathway analyses revealed an enrichment of miR-206 targets to the mTOR pathway, miR-106b targets to the TRIF pathway, and miR-203 targets mapped to cytokine pathways like IL-5. Activation of the mTOR pathway was recently reported in patients experiencing an asthma attack, when compared with patients in asthma remission. This finding could be reproduced in a mouse model of asthma and blockade of the mTOR pathway was shown to ameliorate asthma symptoms (58). miR-106b is a member of the miR-17-92 cluster family, which are all downregulated in Th2 cells. Although all members share almost the same mRNA-targeting seed sequence, only miR-106b was appointed to the top 5 ranking miRs. In vitro profiling experiments of Th1 and Th2 cells had shown an increased expression of the miR-17-92 cluster in Th1 cells, while a suppression was observed in polarized Th2 cells. Active interference with the IL-4 signaling pathway in Th2 cells was reported to induce the expression of this miR family and the authors concluded an inhibitory role of this miR cluster in Th2 polarization (59). We did not test if single members of this cluster would be interchangeable, or if the existing small differences within the family might be decisive. Finally, the miR-203 targets mapped to cytokine pathways important in promoting the Th2 phenotype. This list included IL-5 and IL-3. Silencing this miR might help to form a positive circuit of signals to promote a self-enforcing loop. Taken together, our findings suggest that miRs fine-tune multiple pathways in stable Th2 cells that finally merge into inflammation.

In conclusion, we have systematically profiled $\mathrm{Th} 2$ cells and developed a systems biology tool (IDEAL) to evaluate the role of miRs in controlling persistence of the asthmatic Th2 response. We went beyond the usual expression FC-based analyses and investigated the influence spread of miRs in the molecular interaction network (interactome). We provide evidence and proof for the usefulness of a holistic systems immunology approach to decipher delicate interactions converging to pathological traits and provide proof of a complex and combinatorial activity of a unique set of miRs in Th2 cells. Identification of this collective miR impact may open new possibilities for therapeutic interventions targeting a combination of miRs for treatment of Th2-driven disease.

\section{Methods}

Mice. Female BALB/c mice (Charles River) at 6-8 weeks of age were used for the experiments. Mice were housed under standard conditions with free access to rodent chow and water.

OVA sensitization and challenge. Mice were sensitized by 3 intraperitoneal injections of $10 \mu \mathrm{g}$ OVA (grade VI; Sigma-Aldrich) adsorbed to $1.5 \mathrm{mg}$ aluminum hydroxide (Pierce) dissolved in $200 \mu 1$ phosphate-buffered saline (PBS) at days 1, 14, and 21. OVA aerosol challenges (1\% [wt/v] OVA, grade V, Sigma-Aldrich) were performed for 3 consecutive days (acute) or for 12 weeks with 2 consecutive challenges per week, starting at day 26 and 27.

Measurements of serum immunoglobulins. Blood samples were taken 24 hours after the last aerosol challenge from the tail vein. Total IgE, OVA-specific IgG1, IgE, and IgG2a levels were measured by ELISA (BD Bioscience) in the serum as described previously (60).

Assessment of leukocyte distribution in bronchoalveolar lavage. Bronchoalveolar lavage (BAL) was performed as previously described (60). The total number of leukocytes was determined using a CASY TT cell counter (Schaerfe Systems). To differentiate cell types, cytospins were prepared and stained with Diff-Quik (Merz and Dade AG).

Isolation of $C D 4^{+}$T cells from tissues, FACS, and in vitro polarization. The antibodies and isotype controls used in this study were purchased from BD Biosciences, Biolegend, eBioscience, and MD Biosciences and used at appropriate concentrations. 
Mouse tissues for profiling assays. Spleens were excised and mononuclear cells were isolated using a syringe plunger and $100-\mu \mathrm{m}$ cell strainers. Red blood cells were removed by hypotonic lysis using doubledistilled water. Tonicity was restored by the addition of RPMI 1640 (PAA). The resulting cell suspension was washed twice with PBS and used for the isolation of $\mathrm{CD}^{+} \mathrm{T}$ cells according to the manufacturer's instructions (L3T4; Miltenyi Biotec). Naive CD4 ${ }^{+} \mathrm{T}$ cells were stained for CD4 (clone: RM4-5), CD44 (IM7), and CD62L (mel-14) in FACS buffer ( $0.5 \%$ mouse serum in PBS).

Lungs were perfused via the right heart ventricle with PBS, excised, and digested with collagenase D (2 $\mathrm{mg} / \mathrm{ml}$; Roche) and DNase I ( $40 \mu \mathrm{g} / \mathrm{ml}$; Serva) for 30 minutes at $37^{\circ} \mathrm{C}$. The cell suspension was washed twice with PBS. Erythrocytes were removed by hypotonic lysis (see above). Cells were washed twice with PBS and sieved with $30-\mu \mathrm{m}$ cell strainers (Miltenyi Biotec). $\mathrm{CD}^{+} \mathrm{T}$ cells were enriched using the $\mathrm{CD}^{+} \mathrm{T}$ cell isolation kit according to the manufacturer's instructions (L3T4; Miltenyi Biotec). Cells were stained for CD4 (RM4-5), CD69 (H1.2F3), CXCR3 (CXCR3-173), and ST2 (DJ8).

Sorting of live cells for downstream microarray experiments was performed on a FACSAria III (BD Biosciences) maintained by the Flow Cytometry Core facility at the Medical Faculty of Philipps University Marburg. Live/dead discrimination was performed by including 4',6-diamidino-2-phenylindole (DAPI; 2 $\mu \mathrm{M}$, Biolegend). Cells were sorted into RPMI medium containing 10\% mouse serum (PAA). Cells were washed twice with medium containing 10\% mouse serum, 1\% L-glutamine (PAA), 1\% penicillin/streptomycin (PAA), and stimulated with anti-CD3 (17A2; BD Biosciences) and anti-CD28 (37.51; BD Biosciences) for 8 hours (microarrays and mass spectrometry) or 72 hours (cytokine measurements) at $37^{\circ} \mathrm{C}$ and $5 \% \mathrm{CO}_{2}$. Cell culture supernatants were stored at $-20^{\circ} \mathrm{C}$ until analysis.

Mouse tissues for in vitro analyses. For intracellular cytokine staining, cells were stimulated with phorbol 12-myristate 13-acetate (PMA; $50 \mathrm{ng} / \mathrm{ml}$, eBioscience)/ionomycin ( $1 \mu \mathrm{g} / \mathrm{ml}$, eBioscience) and treated with brefeldin A (eBioscience) for 5 hours. Cells were fixed for 10 minutes with $1 \%$ paraformaldehyde (PFA) in PBS (Roth). Cells were permeabilized using $0.3 \%$ saponin in FACS buffer (0.5\% FBS in PBS). Antibody staining was performed for 30 minutes in the dark using CD4, IFN- $\gamma$ (XMG1.2), IL-9 (RM9A4), IL-10 (JES5-16E3), IL-13 (eBio13A), and IL-17A (eBio17B7). Cells were washed twice with permeabilization buffer and suspended in FACS buffer. For analysis, 20,000 counts (in the CD4 ${ }^{+}$gate) were recorded on a FACSCanto II (BD Biosciences) and analyzed with FlowJo software (Tree Star).

In vitro polarization of mouse Th2 cells. Murine $\mathrm{CD}^{+} \mathrm{CD}^{\mathrm{C}} 2 \mathrm{~L}^{+}$cells were isolated using the Naive $\mathrm{T}$ Cell Isolation Kit II (Miltenyi Biotec) according to the manufacturer's instructions. Cells were activated with plate-bound anti-CD3e (BD Biosciences) and soluble anti-CD28 (BD Biosciences). Th2 cells were polarized in the presence of recombinant murine IL-4 (rmIL-4) $(20 \mathrm{ng} / \mathrm{ml}$; Peprotech), anti-IFN- $\gamma(5 \mu \mathrm{g} / \mathrm{ml}$; BD Biosciences), and anti-IL-12 (5 $\mu \mathrm{g} / \mathrm{ml}$; BD Biosciences) for 3 days. Proliferation of cells was induced using rmIL-2 (20 ng/ml; Peprotech) in the presence of Th2-polarizing reagents for an additional 3 days. Polarization efficiency was verified by staining for intracellular cytokines (please see above).

Total RNA isolation, quality control, and microarray analysis. Total RNA was isolated using peqGOLD TriFast-Reagent (PEQLAB) according to the manufacturer's instructions. Quality of RNA was analyzed using an RNA 6000 Nano Kit on an Agilent Bioanalyzer. Samples were processed according to the Affymetrix manual and hybridized to Affymetrix Mouse Gene 1.0 ST Arrays and Affymetrix GeneChip miRNA 2.0. mRNA and miR microarrays were normalized with robust multiarray average (RMA) through affy followed by t-statistics. Microarray data are archived at the NCBI Gene Expression Omnibus (GEO GSE109737).

Expression data for IL-5 $5^{+}$memory T cells (GSE33516) (31) and CD4 ${ }^{+} \mathrm{T}$ cells isolated from the model of HDM-induced allergic airway inflammation (GSE72005) (32) were retrieved from the GSE database.

$m R N A$ isolation, reverse transcription and real-time RT-PCR. Total RNA from naive $\mathrm{CD}^{+} \mathrm{T}$ cells and Th2 cells was isolated with the RNeasy Mini Kit (Qiagen). First-strand cDNA synthesis was performed with the Omniscript kit (Qiagen). Quantitative real-time PCR was performed with the RotorGene SYBR Green PCR Kit (Qiagen) and a RotorGene6000 System (Qiagen). After an initial denaturation step for 15 minutes at $95^{\circ} \mathrm{C}, 40 \mathrm{PCR}$ cycles of $94^{\circ} \mathrm{C}$ for 15 seconds, $58^{\circ} \mathrm{C}$ for 30 seconds, and $72^{\circ} \mathrm{C}$ for 15 seconds were performed. The primers are mouseCtcf (sense: 5'-GGAAGGACTGCTGTCTGAGG-3', antisense: 5'-CAGATGGCATTTGTGTGGTC-3'), mNcoa3 (sense: 5'-TCAGCGACATCGACAACTTC-3', 5'-AACAGGAAACCATCCAGTGC-3'), mElk3 (sense: 5'-CTCATCTGCTGGACATCGAA-3', 5'-ACAAACTTCTGCCCGATCAC-3'), mAhr (sense: 5'-TTACCTGGGCTTTCAGCAGT-3', antisense: 5'-AGGAAGCTGGTCTGGGGTAT-3'), mElf2 (sense: 5'-TCCTAGCGATGGAGGAGAAA-3', 5'-GCTGTGAACTGATGCTTCCA-3'), and ribosomal L32 (sense: 5'-AAGCGAAACTGGCGGAAACC-3', 
antisense: 5'-CTGGCGTTGGGATTGGTGAC-3') as housekeeping gene. Relative gene expression levels were calculated with the $2^{-\Delta \Delta C t}$ method, with normalization to murine ribosomal L32 as the housekeeping gene. All standard procedures were performed according to the manufacturer's instructions.

Integrated multinetwork - Interactome. While some effort has been devoted to mapping the interactome in mice, at present there is a limited number of high-quality interaction data available $(61,62)$. We therefore used the human interactome combining interactions from the following databases.

(a) $m i R-m R N A$ network: The miR-mRNA network was built using mirDIP, an integration of $12 \mathrm{miR}$ prediction data sets from $6 \mathrm{miR}$ prediction algorithms (63). In order to filter for high-confidence interactions, only interactions that were present in at least 6 prediction datasets were kept, resulting in a bipartite network with 8,374 nodes (542 miRs, 7,832 mRNAs) and 67,517 edges.

(b) Regulatory network: We used the TRANSFAC database (64) that lists experimentally derived regulatory interactions. The resulting network, in which nodes represent transcription factors and connections that are physical binding to regulatory elements, consists of 271 transcription factors and 564 regulated genes. Moreover, tissue-specific regulatory interactions were added from a human regulatory lexicon (65). From the 41 cell-type-specific regulatory networks, the following cell types were considered: human lung microvascular endothelial cells (HMVEC_LLy), human pulmonary fibroblasts (HPFs), human fetal lung (IMR90), normal human lung fibroblasts (NHLFs), human small airway epithelial cells, Th1 T helper cell, lung, CD2, and B lymphocyte (GM06990) in our analysis (65).

(c) Protein-protein interaction network: (i) Binary interactions. We combined several yeast 2-hybrid highthroughput data sets (66-70) with binary interactions from the IntAct (71) and MINT databases (72). The sum of these data sources yielded 28,653 interactions among 8,120 proteins. Of note, IntAct and MINT provide interactions derived from both literature curation and direct submissions. (ii) Literature-curated interactions. These interactions, typically obtained by low-throughput experiments, are manually curated from the literature. We used IntAct (71), MINT (72), BioGRID (73), and HPRD (74), resulting in 74,195 interactions among 10,890 proteins. (iii) Metabolic enzyme-coupled interactions. We assumed that 2 enzymes are coupled if they share adjacent reactions in the KEGG and BIGG databases. In total, we obtained 10,642 metabolic links among 921 enzymes from Lee et al. (75). (iv) Protein complexes. A protein complex is a single molecular unit that integrates multiple gene products. The CORUM database (76) is a collection of mammalian complexes derived from a variety of experimental tools (e.g., coimmunoprecipitation to cosedimentation and ion-exchange chromatography). In total, 2,837 complexes with 2,069 proteins connected by 31,276 links were extracted. (v) Kinase network (kinase-substrate pairs). Protein kinases are important regulators in different biological processes, such as signal transduction. PhosphositePlus (77) provides a network of 327 kinases connected to 1,771 substrates. (vi) Signaling interactions data set provides 32,706 interactions among 6,339 proteins that integrate several sources, both high throughput and literature curation, into a directed network in which cellular signals are transmitted by protein-protein interactions. Note that we do not take the direction of these interactions into account (78).

The union of all interactions obtained yields a multinetwork of 14,237 proteins and 542 miRs that are interconnected by 67,517 miR-mRNA and 206,279 protein-protein interactions.

Differential expression analysis. Both in miR and mRNA cases, log2-transformed values of the expression were averaged over replicates. Let $\mu_{1}$ and $\mu_{2}$ be these $\log 2$ averages in 2 conditions (e.g., naive and stable Th2). The FC is defined as FC $=2^{\mu 2-\mu 1}$. When $\mathrm{FC}<1$, we show the value $-1 / \mathrm{FC}$ for symmetry purposes and an easier reading of the proportion of increase or decrease. The $P$ values were computed using Student's $t$ test on the $\log 2$ values of expression across replicates, and $P<0.05$ was required for defining an mRNA or miR as significantly differentially expressed.

Mouse to human homologs. Mouse gene IDs were converted to human using the Mouse Genome Informatics (MGI) database (http://www.informatics.jax.org/).

IDEAL algorithm. In this work, we developed IDEAL (Impact of Differential Expression Across Layers in multiple omics network) to infer the key miRs whose perturbation is most likely to induce the observed global gene expression changes. IDEAL proceeds in 2 steps: first, the definition of a disease module in the interactome, and second, the prioritization of miRs based on their topological impact on this disease module.

Step 1: Previously, we showed that proteins that agglomerate in specific interactome neighborhoods could potentially be part of a disease module (29). Thus, in step 1, we identify the differential expression threshold above which DE mRNAs significantly cluster in the interactome. To reject the null hypothesis that proteins do not aggregate in the same neighborhood of the interactome, we distributed the same 
number of randomly selected proteins and determined the size of the LCC - the number of nodes in the largest connected subgraph. To determine the significance of the LCC size, we computed the $Z$ score as $Z=\left[L C C-\mu\left(L C C_{r}\right)\right] / \sigma(L C C r)$, where $\mu\left(L C C_{r}\right)$ and $\sigma(L C C r)$ denote the mean and standard deviation, respectively, of the LCC size random expectation.

Step 2: In this step, we introduce the information of the miR-mRNA network. We connect DE miRs with DE genes. Then, based on the expectation that miRs have an inhibitory effect on mRNAs, we only keep edges for which the source miR and target mRNAs show opposite FCs. We then prioritize miRs by their impact on the disease module formed by the DE genes. To that aim, we look for the miR that shows the largest decrease in the LCC relative size (i.e., proportion of genes in the LCC) when removing its targets. We then repeat this process iteratively in an adaptive manner. This defines a ranking for the miRs. We then obtain a curve of LCC relative size as a function of miR rank. In order to assess the significance of the LCC decrease after miR removal, we built a null model to compare to. Beginning from the previously observed subnetwork of DE miRs and mRNAs, we generated 10 randomly shuffled miR-mRNA networks. To do so, we selected a random pool of $\mathrm{miR}$ targets with the same size as in the original network, thereby controlling for miR target overlap. Then, for each miR, we randomly selected the same number of targets as in the original network, thereby controlling for $\mathrm{miR}$ degree. Using these 10 randomly generated networks, we conducted the same adaptive ranking procedure by impact on the LCC and measured the LCC relative size as a function of miR rank. Lastly, we used these random curves to generate a measure of CI of the miRs, defined by their impact strength relative to random. The collective impact $C I$ is defined as the negative of the $Z$ score measuring how the observed fraction of nodes in the LCC departs from the random case: $C I_{i}=-\left[f_{i}-\mu\left(f_{i, r}\right)\right] / \sigma\left(f_{i, r}\right)$, where $f_{i}$ is the fraction of nodes in the LCC for miR of rank $i$, and $\mu\left(f_{i, r}\right)$ and $\sigma\left(f_{i, r}\right)$ denote the mean and standard deviation, respectively, computed from the random networks. This measure is finally used to assess the number of miRs needed to reach maximal impact on the LCC. In this study, we also compare this ranking to the natural ranking by FC (highest to lowest). In that case, the FCs are kept from the original network in the randomized networks.

Pathway enrichment analysis. Genes upregulated in early and stable Th2 cells versus naive T cells (FC $>2 ; P<0.05)$ were subjected to pathway analysis using the Database for Annotation, Visualization and Integrated Discovery (DAVID) $(79,80)$. Identified pathways with $P<0.05$ were considered enriched and are listed in Supplemental tables 1 and 2.

To identify key pathways regulated by the IDEAL miRs, the immediate miR targets in the disease module and their first neighbors were considered to perform pathway enrichment analysis. The Enrich method (81) was applied and only WikiPathways and NCI-Nature pathways in Enrich data source were considered. The identified pathways are listed in Table 1.

Cytokine bead array/Bio-Rad Luminex assay. BAL fluid and cell culture supernatants of FACS-isolated cells were analyzed for the production of IFN- $\gamma$ (limit of detection: $0.5 \mathrm{pg} / \mathrm{ml}), \mathrm{IL}-4$ (0.3 pg/ml), IL-5 (0.9 pg/ml), IL-6 (1.4 pg/ml), IL-9 (10.7 pg/ml), IL-10 (9.6 pg/ml), IL-13 (2.4 pg/ml), and IL-17A (0.95 pg/ $\mathrm{ml}$ ) using Mouse Flex Sets purchased from BD Biosciences on a BD Accuri C6 flow cytometer following the manufacturer's instructions.

Th2 cell supernatants were analyzed 48 hours after nucleofection for the production of IL-5 (0.4 pg/ml), IL-9 (0.6 pg/ml), IL-13 (0.9 pg/ml), and IL-17A (0.6 pg/ml) using Bio-Plex Pro Mouse Cytokine assays on a Bio-Rad (Luminex) Bio-Plex 200 Suspension Array Analyzer following the manufacturer's instructions.

Histological staining. After BAL fluid collection, the right lung was fixed in paraformaldehyde $(6 \%$ $\mathrm{wt} / \mathrm{vol}$ ). Systematic uniform random sampling (SURS) was performed to obtain a representative collection of lung tissue samples (82). After paraffin embedment, 3- $\mu$ m sections were stained with hematoxylin and eosin. Collagen fibrils were detected by means of staining with Sirius Red (Sigma-Aldrich)/Fast Green (Rowley Biochemicals).

Construction and mutagenesis of luciferase expression vectors. WT 3'-UTR sequences of the human CCCTC-binding factor (CTCF), nuclear receptor coactivator 3 (hNCAO3), ELK3 ETS transcription factor (hELK3), aryl hydrocarbon receptor (hAHR), and E74-like ETS transcription factor 2 (hElf2) gene containing the miR binding sites were PCR amplified using HotStarTaq DNA-Polymerase (Qiagen) using human genomic DNA isolated from peripheral blood mononuclear cells and the following primers: humCTCF-UTR WT sense 5'-GCAGAGGGAGAGGAAGAGGA-3'; humCTCF-UTR WT 
antisense; humNCOA3-UTR WT sense 5'-TTGTCCAGTGGAATTGGTGA-3'; humNCOA3-UTR WT antisense 5'-CACACACACAGCCCATGAAT-3'; humELK3-UTR WT sense 5'-CCACGGGCTAGTTTACCTGT-3'; humELK3-UTR WT antisense 5'-CAGGGGAACCCCAACTTACT-3'; humAHRUTR WT sense 5'-TTTTGACCCTGGTTTTTGGA-3'; humAHR-UTR WT antisense 5'-ACTTTGCACCAAACCTTGCT-3'; humELF2-UTR WT sense 5'-AAGGATCGAAAGGGACCAAG-3'; humELF2-UTR WT antisense 5'-ACCTGCCAAAAATTCATGCT-3' using XhoI and XbaI restriction sites. Amplification products were purified using the PCR purification kit (Qiagen), digested with FastDigest enzymes (Thermo Fisher Scientific), and inserted into the XhoI and XbaI sites in the multiple cloning region of the pmirGLO Dual-Luciferase miR Target Expression vector (Promega). Mutations/ deletions of the putative miR-seed sequence were induced using the QuikChange MultiSite-Directed Mutagenesis Kit (Stratagene Technologies) using following primers: humCTCF-UTR $\Delta$ miR-27b sense 5'-AATCATGGAATGTTCTGAGAGTGCTGAGGACAGTGTTG-3'; humCTCF-UTR $\Delta$ miR-27b antisense 5'-CAACACTGTCCTCAGCACTCTCAGAACATTCCATGATT-3'; humNCOA3-UTR $\triangle$ miR-206 sense 5'-GTCTTGAGGGAATAGTGAAACGGTTTTTGCCTACACTTAC-3'; humNCOA3UTR $\triangle$ miR-206 antisense 5'-GTAAGTGTAGGCAAAAACCGTTTCACTATTCCCTCAAGAC-3'; humELK3-UTR $\Delta$ miR-106b sense 5'-GGACATTGTGAAACTCTTGTTAATTTGTCATAACATGGATAGTCTAGATTTAT-3'; humELK3-UTR $\triangle$ miR-106b antisense 5'-ATAAATCTAGACTATCCATGTTATGACAAATTAACAAGAGTTTCACAATGTCC-3'; humAHR-UTR $\Delta$ miR-203 1 sense 5'-GGTGAGGTACCGTCTACATTATTCTGGGCACC-3', humAHR-UTR $\Delta$ miR-203 1 antisense; 5'-GGTGCCCAGAATAATGTAGACGGTACCTCACC-3'; humAHR-UTR $\triangle$ miR-203 2 sense 5'-TCTAGCTTTTTAATTAAGCAAAGCACCAATTGTCTTTAAAAACTGTTTTAGACC-3'; humAHR-UTR $\triangle$ miR-203 2 antisense 5'-GGTCTAAAACAGTTTTTAAAGACAATTGGTGCTTTGCTTAATTAAAAAGCTAGA-3'; humELF2-UTR $\triangle$ miR-23b sense 5'-AGACTCTGCTAATATAGGTATTTTAGATTGAAGAATGGATCCCAAAAA-3'; humELF2-UTR $\triangle$ miR-23b antisense 5'-TTTTTGGGATCCATTCTTCAATCTAAAATACCTATATTAGCAGAGTCT-3'. WT and mutated UTR sequences were verified by DNA sequencing (Seqlab) using the following primers: forward (at 7108-7125 bp of pmiRGlo) 5'-ACACGGTAAAACCATGAC-3' and reverse (at 120-138 bp of pmiRGlo) 5'-GTCCAAACTCATCAATGTA-3'. Lipopolysaccharide-free plasmid was prepared using NucleoBond Xtra plasmid purification kits (Macherey-Nagel).

Transient transfection and reporter gene analysis. 293T cells (ATCC CRL-3216) were seeded at a density of $5 \times 10^{4}$ cells per well (48-well plate) and were grown overnight. Cotransfection of $0.3 \mu \mathrm{g}$ reporter plasmid and $50 \mathrm{nM}$ control miR (miR Mimic Negative Control \#1, Ambion) or $50 \mathrm{nM}$ miR-mimic (hsa-miR-27b3p, MIMAT0000419; hsa-miR-206, MIMAT0000462; hsa-miR-106b-5p, MIMAT0000680; hsa-miR-203a3p, MIMAT0000264; hsa-miR-23b-3p, MIMAT0000418; Ambion and Dharmacon); was performed using Lipofectamine 2000 (Thermo Fisher Scientific). Cell lysates were prepared 24 hours after transfection and luciferase activity was assayed with the Dual Luciferase Reporter Assay kit (Promega) according to the manufacturer's instructions and using the Infinite F200 PRO microplate reader (Tecan). Firefly luciferase activity was normalized to Renilla luciferase activity. Each assay was performed in triplicate and repeated 3 times.

Nucleofection of $T$ cells. Naive CD4 ${ }^{+} \mathrm{CD} 62 \mathrm{~L}^{+} \mathrm{T}$ cells were polarized into Th2 cells for 5 days (see above) and nucleofection was performed with miR mimic and anti-miR inhibitors (mmu-miR-27b-3p, MIMAT0000126; mmu-miR-206-3p, MIMAT0000239; mmu-miR-106b-5p, MIMAT0000386; mmu-miR-203-3p, MIMAT0000236; mmu-miR-23b-3p, MIMAT0000125; all Ambion and $500 \mathrm{nM}$ each) in OptiMEM (Gibco) using the P3 Primary Cell 4D Nucleofector X Kit according to the manufacturer's instructions (Lonza). Transfections were performed in additive fashion antagonizing the predicted cumulative miR effect as predicted by IDEAL. For each condition, an equal amount and respective composition of miR mimics and miR inhibitors was transfected as control sequences to control for sequence specificity (miR Mimic Negative Control \#1 and Anti-miR miR Inhibitor Negative Control \#1; both Ambion). Cells were cultured after nucleofection with plate-bound anti-CD3 and rmIL-2 $(20 \mathrm{ng} / \mathrm{ml})$. The phenotype was evaluated 24 and 48 hours after nucleofection using intracellular cytokine measurements and Luminex Assay (see above).

Statistics. Data analyses were performed using the Prism 6 Software package (GraphPad Software). Data were expressed as mean \pm SEM unless otherwise stated. Where appropriate, a Mann-Whitney $U$ test was done. $P$ values of less than 0.05 were considered statistically significant.

Study approval. All animal experimental procedures were approved by the local animal ethics committee at the Regierungspräsidium Giessen, Germany. 


\section{Author contributions}

AK, M. Santolini, STW, AS, and HR designed the experiments, conducted experiments, analyzed data, and wrote the manuscript. TN, M. Schiller, YP, and MT performed experiments. PG, TB, and SU analyzed data and wrote and critically reviewed the manuscript.

\section{Acknowledgments}

We thank Wilma Hastedt, Nicole Löwer, Thomas Ruppersberg, Alexandra Fischer, Sophia Bernhardt, and the FACS-Core facility of the Philipps University Marburg for excellent technical assistance and Jorun Braun for excellent secretarial assistance. This work was supported by the P.E. Kempkes Foundation (to A. Kilıç), von Behring-Röntgen Foundation (to T. Braun and H. Renz), Universities Giessen and Marburg Lung Centre (UGMLC; to A. Kilıç, H. Renz, S. Uchida, and T. Braun), and the German Center for Lung Research (DZL Disease Area Allergy and Asthma; to H. Renz).

Address correspondence to: Harald Renz, Institute of Laboratory Medicine, Philipps University Marburg, 35043 Marburg, Germany. Phone: 49.6421.586.6235; Email: harald.renz@uk-gm.de.

1. Holgate ST, Wenzel S, Postma DS, Weiss ST, Renz H, Sly PD. Asthma. Nat Rev Dis Primers. 2015;1:15025.

2. Wenzel SE. Asthma phenotypes: the evolution from clinical to molecular approaches. Nat Med. 2012;18(5):716-725.

3. Holgate ST. Stratified approaches to the treatment of asthma. Br J Clin Pharmacol. 2013;76(2):277-291.

4. Campo P, et al. Phenotypes and endotypes of uncontrolled severe asthma: new treatments. J Investig Allergol Clin Immunol. 2013;23(2):76-88.

5. Ansel KM, Djuretic I, Tanasa B, Rao A. Regulation of Th2 differentiation and I14 locus accessibility. Annu Rev Immunol. 2006;24:607-656.

6. Zhu J, Yamane H, Paul WE. Differentiation of effector CD4 T cell populations $\left(^{*}\right)$. Annu Rev Immunol. 2010;28:445-489.

7. Littman DR, Rudensky AY. Th17 and regulatory T cells in mediating and restraining inflammation. Cell. 2010;140(6):845-858.

8. Purwar R, et al. Robust tumor immunity to melanoma mediated by interleukin-9-producing T cells. Nat Med. 2012;18(8):1248-1253.

9. Veldhoen M, et al. Transforming growth factor-beta 'reprograms' the differentiation of T helper 2 cells and promotes an interleukin 9-producing subset. Nat Immunol. 2008;9(12):1341-1346.

10. Chung KF. Targeting the interleukin pathway in the treatment of asthma. Lancet. 2015;386(9998):1086-1096.

11. Fahy JV. Type 2 inflammation in asthma--present in most, absent in many. Nat Rev Immunol. 2015;15(1):57-65.

12. Paul WE, Zhu J. How are T(H)2-type immune responses initiated and amplified? Nat Rev Immunol. 2010;10(4):225-235.

13. O'Connell RM, Rao DS, Chaudhuri AA, Baltimore D. Physiological and pathological roles for microRNAs in the immune system. Nat Rev Immunol. 2010;10(2):111-122.

14. Liston A, Linterman M, Lu LF. MicroRNA in the adaptive immune system, in sickness and in health. J Clin Immunol. 2010;30(3):339-346.

15. Rebane A, Akdis CA. MicroRNAs in allergy and asthma. Curr Allergy Asthma Rep. 2014;14(4):424.

16. Bartel DP. MicroRNAs: target recognition and regulatory functions. Cell. 2009;136(2):215-233.

17. Kim VN, Han J, Siomi MC. Biogenesis of small RNAs in animals. Nat Rev Mol Cell Biol. 2009;10(2):126-139.

18. Rebane A, Akdis CA. MicroRNAs: Essential players in the regulation of inflammation. J Allergy Clin Immunol. 2013;132(1):15-26.

19. Singh Y, et al. Mycobacterium tuberculosis controls microRNA-99b (miR-99b) expression in infected murine dendritic cells to modulate host immunity. J Biol Chem. 2013;288(7):5056-5061.

20. Simpson LJ, et al. A microRNA upregulated in asthma airway T cells promotes TH2 cytokine production. Nat Immunol. 2014;15(12):1162-1170.

21. Pua HH, et al. MicroRNAs 24 and 27 suppress allergic inflammation and target a network of regulators of $\mathrm{T}$ helper 2 cellassociated cytokine production. Immunity. 2016;44(4):821-832.

22. Kim RY, et al. MicroRNA-21 drives severe, steroid-insensitive experimental asthma by amplifying phosphoinositide 3-kinasemediated suppression of histone deacetylase 2. J Allergy Clin Immunol. 2017;139(2):519-532.

23. Xu J, et al. Prioritizing candidate disease miRNAs by topological features in the miRNA target-dysregulated network: case study of prostate cancer. Mol Cancer Ther. 2011;10(10):1857-1866.

24. Gu C, Liao B, Li X, Li K. Network consistency projection for human miRNA-disease associations inference. Sci Rep. 2016;6:36054.

25. Nunez YO, et al. Positively correlated miRNA-mRNA regulatory networks in mouse frontal cortex during early stages of alcohol dependence. BMC Genomics. 2013;14:725.

26. Liu Y, et al. MiR-138 suppresses airway smooth muscle cell proliferation through the PI3K/AKT signaling pathway by targeting PDK1. Exp Lung Res. 2015;41(7):363-369.

27. Kitsak M, et al. Tissue specificity of human disease module. Sci Rep. 2016;6:35241

28. Menche J, et al. A diVIsive Shuffling Approach (VIStA) for gene expression analysis to identify subtypes in chronic obstructive pulmonary disease. BMC Syst Biol. 2014;8 Suppl 2:S8.

29. Menche J, et al. Disease networks. Uncovering disease-disease relationships through the incomplete interactome. Science. 2015;347(6224):1257601.

30. Sharma A, et al. A disease module in the interactome explains disease heterogeneity, drug response and captures novel pathways and genes in asthma. Hum Mol Genet. 2015;24(11):3005-3020. 
31. Endo Y, et al. Eomesodermin controls interleukin-5 production in memory T helper 2 cells through inhibition of activity of the transcription factor GATA3. Immunity. 2011;35(5):733-745.

32. Coquet JM, et al. Interleukin-21-producing CD4(+) T cells promote type 2 immunity to house dust mites. Immunity. 2015;43(2):318-330

33. Mikami N, et al. Calcitonin gene-related peptide is an important regulator of cutaneous immunity: effect on dendritic cell and T cell functions. J Immunol. 2011;186(12):6886-6893

34. Rochlitzer S, et al. The neuropeptide calcitonin gene-related peptide affects allergic airway inflammation by modulating dendritic cell function. Clin Exp Allergy. 2011;41(11):1609-1621.

35. Bronevetsky Y, et al. T cell activation induces proteasomal degradation of Argonaute and rapid remodeling of the microRNA repertoire. J Exp Med. 2013;210(2):417-432.

36. Kotlyar M, Pastrello C, Sheahan N, Jurisica I. Integrated interactions database: tissue-specific view of the human and model organism interactomes. Nucleic Acids Res. 2016;44(D1):D536-D541.

37. Pérez-Bercoff $\AA$, Hudson CM, Conant GC. A conserved mammalian protein interaction network. PLoS ONE. 2013;8(1):e52581.

38. Albert R, Jeong H, Barabasi AL. Error and attack tolerance of complex networks. Nature. 2000;406(6794):378-382.

39. Mills KH. Regulatory T cells: friend or foe in immunity to infection? Nat Rev Immunol. 2004;4(11):841-855.

40. Zaiss DM, Yang L, Shah PR, Kobie JJ, Urban JF, Mosmann TR. Amphiregulin, a TH2 cytokine enhancing resistance to nematodes. Science. 2006;314(5806):1746.

41. Delgoffe GM, et al. The mTOR kinase differentially regulates effector and regulatory T cell lineage commitment. Immunity. 2009;30(6):832-844

42. Xiao C, et al. Lymphoproliferative disease and autoimmunity in mice with increased miR-17-92 expression in lymphocytes. Nat Immunol. 2008;9(4):405-414.

43. Sonkoly E, et al. MicroRNAs: novel regulators involved in the pathogenesis of psoriasis? PLoS One. 2007;2(7):e610.

44. Kubo M, Hanada T, Yoshimura A. Suppressors of cytokine signaling and immunity. Nat Immunol. 2003;4(12):1169-1176

45. Kim HK, Lee YS, Sivaprasad U, Malhotra A, Dutta A. Muscle-specific microRNA miR-206 promotes muscle differentiation. J Cell Biol. 2006;174(5):677-687.

46. Baek D, Villén J, Shin C, Camargo FD, Gygi SP, Bartel DP. The impact of microRNAs on protein output. Nature. 2008;455(7209):64-71

47. Bracken CP, Scott HS, Goodall GJ. A network-biology perspective of microRNA function and dysfunction in cancer. Nat Rev Genet. 2016;17(12):719-732.

48. Barabási AL, Gulbahce N, Loscalzo J. Network medicine: a network-based approach to human disease. Nat Rev Genet. 2011;12(1):56-68.

49. Neilson JR, Zheng GX, Burge CB, Sharp PA. Dynamic regulation of miRNA expression in ordered stages of cellular development. Genes Dev. 2007;21(5):578-589.

50. Wang H, et al. miR-422a Inhibits Glioma Proliferation and Invasion by Targeting IGF1 and IGF1R. Oncol Res. 2017;25(2):187-194.

51. Wang $\mathrm{P}$, et al. Identification of resting and type I IFN-activated human NK cell miRNomes reveals microRNA-378 and microRNA-30e as negative regulators of NK cell cytotoxicity. J Immunol. 2012;189(1):211-221.

52. Perry MM, Baker JE, Gibeon DS, Adcock IM, Chung KF. Airway smooth muscle hyperproliferation is regulated by microRNA-221 in severe asthma. Am J Respir Cell Mol Biol. 2014;50(1):7-17.

53. Mattes J, Collison A, Plank M, Phipps S, Foster PS. Antagonism of microRNA-126 suppresses the effector function of TH2 cells and the development of allergic airways disease. Proc Natl Acad Sci USA. 2009;106(44):18704-18709.

54. Malmhäll C, et al. MicroRNA-155 is essential for T(H)2-mediated allergen-induced eosinophilic inflammation in the lung. $J$ Allergy Clin Immunol. 2014;133(5):1429-1438.

55. Plank MW, et al. MicroRNA expression is altered in an ovalbumin-induced asthma model and targeting miR-155 with antagomirs reveals cellular specificity. PLoS One. 2015;10(12):e0144810

56. Cho S, et al. miR-23 27 24 clusters control effector T cell differentiation and function. J Exp Med. 2016;213(2):235-249.

57. Cruz LO, et al. Excessive expression of miR-27 impairs Treg-mediated immunological tolerance. J Clin Invest. 2017;127(2):530-542.

58. Zhang Y, et al. Activation of the mTOR signaling pathway is required for asthma onset. Sci Rep. 2017;7(1):4532

59. Sasaki K, et al. miR-17-92 expression in differentiated T cells - implications for cancer immunotherapy. J Transl Med. $2010 ; 8: 17$.

60. Renz H, Smith HR, Henson JE, Ray BS, Irvin CG, Gelfand EW. Aerosolized antigen exposure without adjuvant causes increased IgE production and increased airway responsiveness in the mouse. J Allergy Clin Immunol. 1992;89(6):1127-1138.

61. Xia K, Dong D, Han JD. IntNetDB v1.0: an integrated protein-protein interaction network database generated by a probabilistic model. BMC Bioinformatics. 2006;7:508

62. Wiles AM, et al. Building and analyzing protein interactome networks by cross-species comparisons. BMC Syst Biol. 2010;4:36

63. Shirdel EA, Xie W, Mak TW, Jurisica I. NAViGaTing the micronome--using multiple microRNA prediction databases to identify signalling pathway-associated microRNAs. PLoS One. 2011;6(2):e17429.

64. Wingender E. The TRANSFAC project as an example of framework technology that supports the analysis of genomic regulation. Brief Bioinformatics. 2008;9(4):326-332.

65. Neph S, et al. An expansive human regulatory lexicon encoded in transcription factor footprints. Nature. 2012;489(7414):83-90. 66. Stelzl U, et al. A human protein-protein interaction network: a resource for annotating the proteome. Cell. 2005;122(6):957-968 67. Yu H, et al. Next-generation sequencing to generate interactome datasets. Nat Methods. 2011;8(6):478-480.

68. Venkatesan K, et al. An empirical framework for binary interactome mapping. Nat Methods. 2009;6(1):83-90.

69. Rual JF, et al. Towards a proteome-scale map of the human protein-protein interaction network. Nature. 2005;437(7062):1173-1178.

70. Human Reference Protein Interactome Project. Center for Cancer Systems Biology. http://interactome.baderlab.org/. Accessed April 10, 2018.

71. Aranda B, et al. The IntAct molecular interaction database in 2010. Nucleic Acids Res. 2010;38(Database issue):D525-D531.

72. Ceol A, et al. MINT, the molecular interaction database: 2009 update. Nucleic Acids Res. 2010;38(Database issue):D532-D539.

73. Stark C, et al. The BioGRID interaction database: 2011 update. Nucleic Acids Res. 2011;39(Database issue):D698-D704.

74. Keshava Prasad TS, et al. Human Protein Reference Database--2009 update. Nucleic Acids Res. 2009;37(Database issue):D767-D772. 
75. Lee DS, Park J, Kay KA, Christakis NA, Oltvai ZN, Barabási AL. The implications of human metabolic network topology for disease comorbidity. Proc Natl Acad Sci USA. 2008;105(29):9880-9885.

76. Ruepp A, et al. CORUM: the comprehensive resource of mammalian protein complexes--2009. Nucleic Acids Res. 2010;38(Database issue):D497-D501.

77. Hornbeck PV, et al. PhosphoSitePlus: a comprehensive resource for investigating the structure and function of experimentally determined post-translational modifications in man and mouse. Nucleic Acids Res. 2012;40(Database issue):D261-D270.

78. Vinayagam A, et al. A directed protein interaction network for investigating intracellular signal transduction. Sci Signal. 2011;4(189):rs8.

79. Huang da W, Sherman BT, Lempicki RA. Bioinformatics enrichment tools: paths toward the comprehensive functional analysis of large gene lists. Nucleic Acids Res. 2009;37(1):1-13.

80. Huang da W, Sherman BT, Lempicki RA. Systematic and integrative analysis of large gene lists using DAVID bioinformatics resources. Nat Protoc. 2009;4(1):44-57.

81. Kuleshov MV, et al. Enrichr: a comprehensive gene set enrichment analysis web server 2016 update. Nucleic Acids Res. 2016;44(W1):W90-W97.

82. Yildirim AO, et al. Keratinocyte growth factor protects against Clara cell injury induced by naphthalene. Eur Respir J. 2008;32(3):694-704. 